Draft VERSion SEPTEMBer 29, 2018

Preprint typeset using $\mathrm{LAT}_{\mathrm{E}} \mathrm{X}$ style emulateapj v. 8/13/10

\title{
CONNECTION BETWEEN THE ACCRETION DISK AND JET IN THE RADIO GALAXY 3C 111
}

\author{
Ritaban Chatterjee ${ }^{1}$, Alan P. Marscher ${ }^{2}$, Svetlana G. Jorstad ${ }^{2,3}$, Alex Markowitz ${ }^{4}$, Elizabeth Rivers ${ }^{4}$, \\ Richard E. Rothinchild ${ }^{4}$, Ian M. Mchardy ${ }^{5}$, Margo F. Aller ${ }^{6}$, Hugh D. Aller ${ }^{6}$, Anne Lähteenmäki ${ }^{7}$, Merja \\ Tornikoski ${ }^{7}$, Brandon Harrison ${ }^{2}$, Iván AGudo ${ }^{2,8}$, José L. Gómez ${ }^{8}$, Brian W. Taylor ${ }^{2,9}$, Mark Gurwell ${ }^{10}$ \\ Draft version September 29, 2018
}

\begin{abstract}
We present the results of extensive multi-frequency monitoring of the radio galaxy $3 \mathrm{C} 111$ between 2004 and 2010 at X-ray (2.4-10 keV), optical ( $R$ band), and radio (14.5, 37, and $230 \mathrm{GHz}$ ) wave bands, as well as multi-epoch imaging with the Very Long Baseline Array (VLBA) at $43 \mathrm{GHz}$. Over the six years of observation, significant dips in the X-ray light curve are followed by ejections of bright superluminal knots in the VLBA images. This shows a clear connection between the radiative state near the black hole, where the X-rays are produced, and events in the jet. The X-ray continuum flux and Fe line intensity are strongly correlated, with a time lag shorter than 90 days and consistent with zero. This implies that the Fe line is generated within 90 light-days of the source of the X-ray continuum. The power spectral density function of X-ray variations contains a break, with steeper slope at shorter timescales. The break timescale of $13_{-6}^{+12}$ days is commensurate with scaling according to the mass of the central black hole based on observations of Seyfert galaxies and black hole X-ray binaries (BHXRBs). The data are consistent with the standard paradigm, in which the X-rays are predominantly produced by inverse Compton scattering of thermal optical/UV seed photons from the accretion disk by a distribution of hot electrons - the corona - situated near the disk. Most of the optical emission is generated in the accretion disk due to reprocessing of the X-ray emission. The relationships that we have uncovered between the accretion disk and the jet in 3C 111, as well as in the FR I radio galaxy 3C 120 in a previous paper, support the paradigm that active galactic nuclei and Galactic BHXRBs are fundamentally similar, with characteristic time and size scales proportional to the mass of the central black hole.
\end{abstract}

Subject headings: galaxies: active - X-rays: galaxies - X-rays: binaries — Galaxies: individual (3C 111) - quasars: general — Black hole physics

\section{INTRODUCTION}

In many cosmic systems containing jets, e.g., active galactic nuclei (AGNs), stellar mass black hole $\mathrm{X}$ ray binaries (BHXRBs), and young stellar objects, the presence of an accretion disk is inferred from observations. One of the prominent theories of jet production asserts that the jet plasma is propelled by magnetic field lines that thread the accretion disk (and perhaps, in AGNs and BHXRBs, the black hole) and

\footnotetext{
${ }^{1}$ Department of Astronomy, Yale University, PO Box 208101, New Haven, CT 06520-8101; ritaban.chatterjee@yale.edu

${ }^{2}$ Institute for Astrophysical Research, Boston University, 725 Commonwealth Avenue, Boston, MA 02215

${ }^{3}$ Astronomical Institute of St. Petersburg State University, Universitetskij Pr. 28, Petrodvorets, 198504 St. Petersburg, Russia

${ }^{4}$ Center for Astrophysics and Space Sciences, University of California, San Diego, M.C. 0424, La Jolla, CA, 92093-0424, USA

${ }^{5}$ Department of Physics and Astronomy, University of Southampton, Southampton, SO17 1BJ, United Kingdom

${ }_{6}$ Astronomy Department, University of Michigan, 830 Dennison, 501 East University Street, Ann Arbor, Michigan 48109-1042

${ }^{7}$ Aalto University, Metsähovi Radio Observatory, Metsähovintie 114, FIN-02540, Kylmälä, Finland

${ }^{8}$ Instituto de Astrofisica de Andalucia, CSIC, Apartado 3004, 18080 Granada, Spain

${ }^{9}$ Lowell Observatory, 1400 West Mars Hill Road, Flagstaff, AZ., USA

${ }^{10}$ Harvard-Smithsonian Center for Astrophysics, MS 42, 60 Garden Street, Cambridge, MA 02138, USA
}

are twisted by differential rotation (Blandford \& Pavne 1982; Lovelace, Berk \& Contopoulos 1991; Begelman 1995; Meier, Koide \& Uchida 2001; Vlahakis \& König] 2004). This suggests that any relations observed between events in the accretion disk and those in the jet can be used to characterize the disk-jet connection and therefore to constrain models of jet formation. In BHXRBs, the connection between accretion state and events in the jet is well-established. In these objects, transitions to high-soft X-ray states are associated with the emergence of very bright features that proceed to propagate down the radio jet (Fender. Belloni \& Gallo 2004; Fender, Homan \& Belloni 2009).

Observations related to the disk-jet connection in AGNs are complicated, since a single AGN usually does not show the entire range of properties that we wish to analyze. For example, in Seyfert galaxies, most of the observed X-ray (and optical) emission is generated in the accretion disk-corona region, but their radio jets tend to be weak and non-relativistic (e.g., Ulvestad et al. 1999). On the other hand, in radio-loud AGNs with strong, highly variable nonthermal radiation (blazars), the Doppler beamed emission from the jet at most wavelengths masks the thermal emission from the accretion disk and its nearby regions. The radio galaxies 3C 120 and 3C 111 provide an excellent opportunity to study the relationship between events in the accretion disk and those in the jet. At optical and X-ray frequencies, their properties are similar to Seyfert galaxies, e.g., the pres- 
ence of strong broad emission lines in the optical band (Sargent 1977), and an iron emission line in the X-ray spectrum (Lewis et al. 2005), while at centimeter and millimeter wavelengths their emission is dominated by a bright relativistic jet. Nevertheless, the source of the optical continuum emission in radio galaxies is not wellknown. For example, it may be blackbody emission from the accretion disk (Malkan 1983) or synchrotron radiation from the jet.

An accretion disk-jet connection in AGNs has been suggested by Marscher et al. (2002). During $\sim 3$ yr of monitoring of the radio galaxy 3C 120 (1997 to 2000), each of four dips in the X-ray flux, accompanied by spectral hardening, preceded the appearance of a bright knot moving down the radio jet at a superluminal apparent speed. Using similar, but more extensive, multifrequency monitoring observations of this object between 2002 and 2007 at X-ray $(2.4-10 \mathrm{keV})$ and radio (37 $\mathrm{GHz}$ ) wave bands as well as VLBA images at $43 \mathrm{GHz}$, Chatteriee et al. (2009) established an unambiguous connection between events in the accretion disk and jet. They showed that, during this interval, the X-ray and $37 \mathrm{GHz}$ variations are anti-correlated, with the X-ray variations leading those in the radio by $\sim 120$ days. Furthermore, nearly every X-ray dip is followed by the ejection of a new knot in the VLBA images. These findings imply that a decrease in the X-ray production is linked with increased speed in the jet flow, causing a shock wave to form and propagate downstream, appearing as a knot of bright emission in the jet. In $3 \mathrm{C} 120$, the presence of an X-ray $\mathrm{Fe}$ emission line implies that most of the $\mathrm{X}$-rays are produced in the accretion disk region. Therefore, the above pattern is strong evidence for a disk-jet connection.

In this work, we present the results of extensive multifrequency monitoring of 3C 111 between 2004 and 2009 at X-ray energies $(2.4-10 \mathrm{keV})$, optical $R$ band, and radio frequencies 14.5, 37, and $230 \mathrm{GHz}$, as well as imaging with the Very Long Baseline Array (VLBA) at 43 $\mathrm{GHz}$. We use these data to investigate the presence of a disk-jet connection similar to that in 3C 120 . 3C 111 is a relatively nearby $(z=0.049)$ broad-line radio galaxy (BLRG) (Sargent 1977; Lewis et al. 2005). It is classified as a Fanaroff-Riley class II (FR II) radio galaxy (Fanaroff \& Riley 1974) containing two radio lobes with hot spots and a single-sided jet (Linfield \& Perley 1984). At radio frequencies, 3C 111 has blazar-like behavior, although the jet lies at an angle $\sim 18^{\circ}$ to our line of sight (Jorstad et al. 2005), significantly greater than is the case for typical blazars. The counter-jet is too faint to detect, presumably because of Doppler de-boosting. $3 \mathrm{C} 111$ exhibits the brightest compact radio core at $\mathrm{cm} / \mathrm{mm}$ wavelengths of all $\mathrm{FR}$ II radio galaxies and a blazar-like spectral energy distribution (Kadler et al. 2008; Sguera et al. 2005). It was one of the first radio galaxies in which superluminal motion was detected (Götz et al. 1987; Preuss, Alef, \& Kellermann 1987); knots are ejected 1-2 times per year with typical apparent speeds of 3-5c (Jorstad et al. 2005). Recently, Hartman. Kadler \& Tueller (2008) showed that part of the $\gamma$-ray emission from the EGRET source $3 \mathrm{EG} \mathrm{J} 0416+3650$ is associated with $3 \mathrm{C} 111$, and integration over the first 11 months of observations with the Large Area Telescope of the Fermi Space Tele- scope detected 3C 111 at a rather low flux level, $1.5 \pm$ $0.5 \times 10^{-9}$ phot $\mathrm{cm}^{-2} \mathrm{~s}^{-1}$ at energies exceeding $1 \mathrm{GeV}$ (Abdo et al. 2010). At optical and X-ray frequencies, 3C 111 possesses properties similar to Seyfert galaxies and BHXRBs. It has a prominent iron emission line at a rest energy of $6.4 \mathrm{keV}$ (e.g., Revnolds et al. 1998; Eracleous, Sambruna \& Mushotzsky 2000). This implies that most of the X-rays are produced in the immediate environs of the accretion disk: the corona, a hot wind, or the base of the jet (Haardt, Maraschi \& Ghisellini 1994; Markoff, Nowak, \& Wilms 2005; Nayakshin 2000).

Power spectral density (PSD) analysis is a common technique to characterize time variability, which in turn is a powerful diagnostic of the geometry and physics of the accretion disk, corona, and jet. The PSD corresponds to the power in the variability of emission as a function of timescale. Marshall et al. (2009) and Chatteriee et al. (2009) found that the X-ray PSD of 3C 120 can be fit by a piece-wise power law, with a slope that steepens above a break frequency. This property of 3C 120 is similar to Seyfert galaxies and BHXRBs, although in many cases the PSDs of the latter have more than one break, as well as sharp peaks representing quasi-periodic oscillations. The PSD break frequency in BHXRBs and Seyfert galaxies scales inversely with the mass of the black hole (Edelson \& Nandra 1999; Nowak et al. 1999; Pounds et al. 2001; Uttley, McHardy \& Papadakis 2002; Markowitz et al. 2003; McHardy et al. 2004). McHardy et al. (2006) showed that the break frequency and $\mathrm{BH}$ mass are inversely related over a range of $\mathrm{BH}$ masses from 10 to $10^{8} M_{\odot}$, and that, for a given black hole mass, the break frequency also increases with higher accretion rate $(\dot{m})$.

Here, we use our data set to verify that the X-ray PSD of 3C 111 also has a break. Measuring the PSD of $3 \mathrm{C} 111$ gives us an opportunity to test whether another radio-loud AGN (in addition to 3C 120) has a broadband X-ray PSD similar to those of other Seyfert galaxies and BHXRBs. We can investigate whether the break timescale $\left(T_{\mathrm{B}}\right)$ is consistent with the empirical relation between $T_{\mathrm{B}}$, black hole mass $\left(M_{B H}\right)$, and bolometric luminosity $\left(L_{\mathrm{bol}}\right)$ proposed by McHardy et al. (2006), based on a sample of mainly radio-quiet Seyfert galaxies. We analyze the X-ray and radio light curves as well as the sequence of VLBA images to test whether the X-ray dips and ejection of new radio knots in the pc scale jet are temporally related, as is the case in 3C 120 . In order to investigate the source of optical emission in $3 \mathrm{C} 111$, we cross-correlate the optical flux variations with those at X-ray energies. Uneven sampling, as invariably occurs, can cause the correlation coefficients to be artificially low. In addition, the time lags can vary across the years owing to physical changes in the source. In light of these issues, we estimate the significance of the derived correlation coefficients by repeating the analysis with simulated light curves, based on the underlying PSD. We also follow the variation in the degree of polarization of the optical emission in order to discriminate between nonthermal and thermal emission.

In $\S 2$ we present the observations and data reduction procedures, while in $\S 3$ we describe the correlated variations in the X-ray continuum and $\mathrm{Fe} \mathrm{K} \alpha$ emission line. In $\S 4$ we carry out the power spectral analysis and discuss its results and implications. In $\S 5$ we investigate 
Table 1

Parameters of the Light Curves.

\begin{tabular}{ccccccc}
\hline \hline & Data set & Start & End & $\mathrm{T}(\text { days })^{\mathrm{a}}$ & $\Delta \mathrm{T}$ (days) ${ }^{\mathrm{b}}$ & $\mathrm{N}_{\text {points }}{ }^{\mathrm{c}}$ \\
\hline \multirow{3}{*}{$\mathrm{X}-\mathrm{ray}$} & Monitor & 2004 March & 2010 April & 2246.0 & 15.0 & 822 \\
& Medium & 2006 November & 2007 January & 56.0 & 0.25 & 224 \\
& Longlook & 2009 February 16 & 2009 February 17 & 1.2 & 0.01 & 120 \\
\hline Optical & Monitor & 2004 November & 2010 August & 2080.0 & 15.0 & 336 \\
& Medium & 2005 July & 2006 January & 160.0 & 5.0 & 37 \\
\hline $14.5 \mathrm{GHz}$ & Monitor & 2004 February & 2010 February & 2216.0 & - & 398 \\
$37 \mathrm{GHz}$ & Monitor & 2005 January & 2008 December & 1458.0 & - & 98 \\
$230 \mathrm{GHz}$ & Monitor & 2004 January & 2010 April & 2288.0 & - & 161 \\
\hline
\end{tabular}

a Total length of light curves.

b Bin size.

c Number of data points in the unbinned light curve.

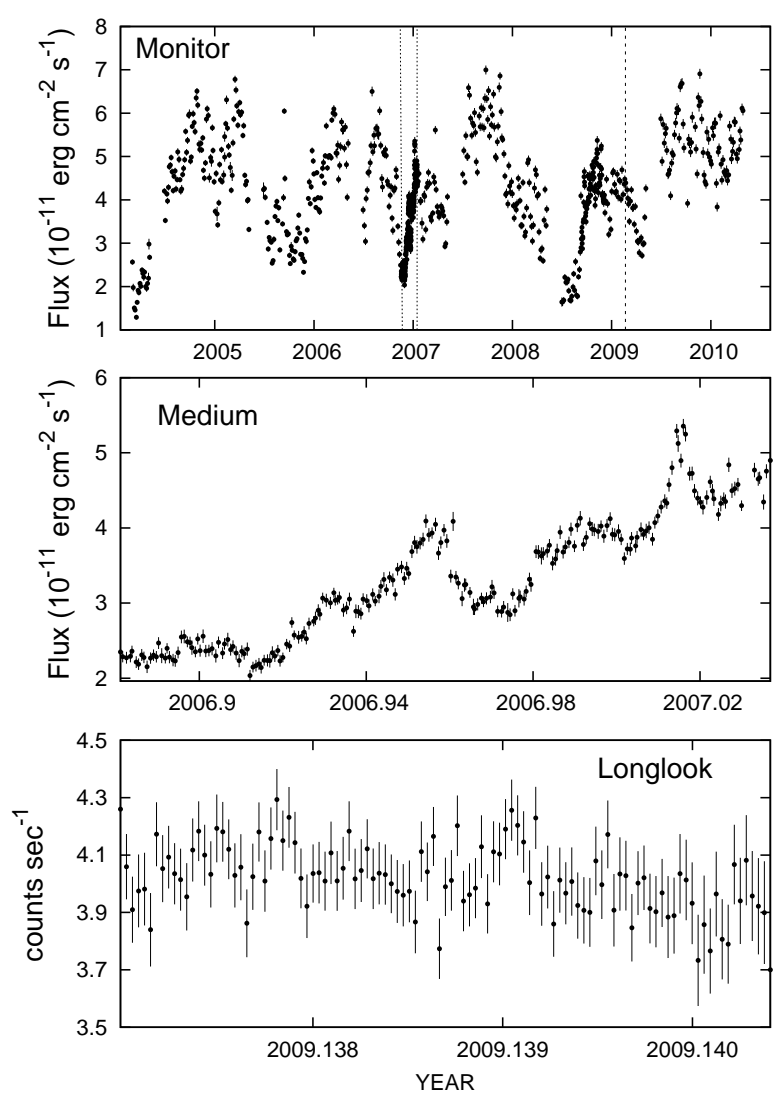

Figure 1. X-ray light curves of 3C 111 with different sampling rates. The dotted and dashed vertical lines at the top panel denote the intervals shown in the middle and bottom panel, respectively.

the relation between the X-ray dips and ejections of superluminal radio knots, as well as the X-ray/radio flux correlation. In $\S 6$ we cross-correlate the X-ray and optical variations and discuss the possible sources of optical emission. In $\S 7$ we compare the properties of $3 \mathrm{C} 111$ with those of the radio galaxy $3 \mathrm{C} 120$, while $\S 8$ contains the summary and conclusions.

\section{OBSERVATIONS AND DATA ANALYSIS}

Table 1 summarizes the intervals of monitoring at different frequencies for each of the three wave bands in our data set. We term the entire light curve "monitor data"; shorter segments of more intense monitoring are described below.

\subsection{X-Ray Monitoring}

The X-ray light curves are based on observations of 3C 111 with the PCA detector of the Rossi X-ray Timing Explorer (RXTE) from 2004 March to 2010 April, with typical exposure times of 1-2 ks. For each exposure, we used routines from the X-ray data analysis software FTOOLS and the program XSPEC version 11.6 to calculate and subtract the standard Xray background model for the faint sources from the data and to fit the X-ray spectrum from 2.4 to $10 \mathrm{keV}$ as a power law with low-energy photoelectric absorption by intervening gas in our Galaxy. For the latter, we used a hydrogen column density of $9.0 \times 10^{21}$ atoms $\mathrm{cm}^{-2}$. This value of the absorbing column was determined by Sambruna, Eracleous \& Mushotzky (1999) by analyzing the X-ray spectrum of 3C 111 with data from the Advanced Satellite for Cosmology and Astrophysics (ASCA). Using observations from $X M M-N e w t o n$, Lewis et al. (2005) have also obtained a value close to this. It should be noted that a giant molecular cloud is present in the foreground of $3 \mathrm{C}$ 111 (Marscher. Moore \& Bania 1993; Moore \& Marscher 1995) and it contributes significantly to the optical to Xray extinction of emission received from $3 \mathrm{C} 111$.

The sampling of the X-ray flux varied. Normally, observations were made 2-3 times per week except during 8 -week intervals each year when the radio galaxy is too close to the Sun's celestial position to observe safely. In order to sample shorter-term variations, between 2006 November and 2007 January we obtained, on average, four measurements per day for almost two months. We refer to these observations as the "medium" data. Our monitoring program also contains an interval from 2008 September to 2008 December when we obtained roughly one data point per day. In order to sample variations on as short timescales as possible, we observed 3C 111 with the European Photon Imaging Camera (EPIC) on board XMM-Newton continuously for about $130 \mathrm{ks}$ on 2009 February 16-17. The EPIC-pn and EPIC-MOS data were obtained in the Small Window and Partial Window mode, respectively, using the thin filter. The data were processed with the the Science Analysis Software version 8.0.0. We filtered the pn data to include only single and double-pixel events (i.e., PATTERN $\leq$ 4). A pn light curve was extracted in the $2.4-10 \mathrm{keV}$ energy band, similar to that of our RXTE data, and was 


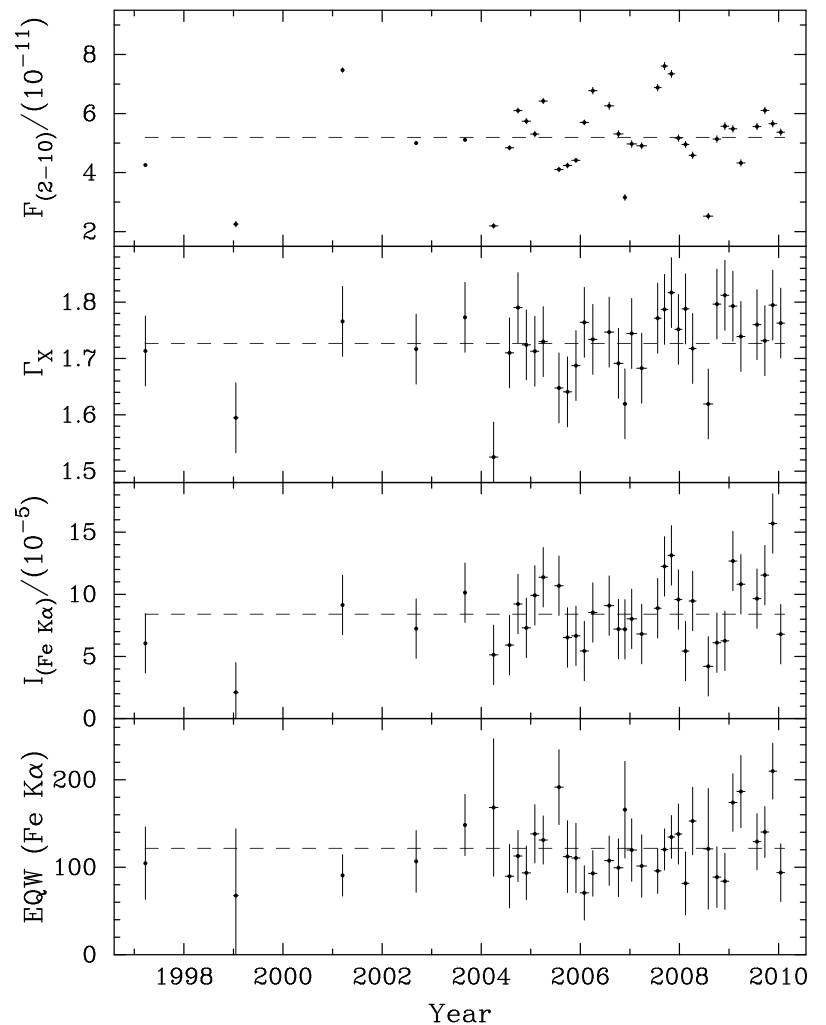

Figure 2. Light curves for $2.4-10 \mathrm{keV}$ continuum flux $F_{2-10}$ (in erg $\mathrm{cm}^{-2} \mathrm{~s}^{-1}$ ), photon index $\Gamma_{\mathrm{X}}$ (of the continuum), intensity of the Fe line $I_{\mathrm{FeK} \alpha}$ (in $\mathrm{ph} \mathrm{cm}^{-2} \mathrm{~s}^{-1}$ ), and the Fe line equivalent width $E Q W$ (in eV) derived from time-resolved spectral fits to the $R X T E$-PCA monitoring data. The dashed lines are the mean values.

background-subtracted and binned to $1000 \mathrm{~s}$ time intervals. In general, pn data are more suitable for timing analysis and the average count rate in the pn light curve was higher than that in the MOS light curves. Hence, we use the pn light curve as the "longlook" data. Figure 1 presents these three data sets. The X-ray data are given in Tables 2 and 3 of the electronic version.

We performed time-resolved spectroscopy on the $R X T E$ monitoring data in order to explore the variability of the $\mathrm{Fe} \mathrm{K} \alpha$ emission line. If the line does indeed respond to X-ray continuum variations, then one can place constraints on the distance between the X-ray continuum source and the bulk of the lineemitting gas. The analysis closely followed that employed by previous studies (e.g., Vaughan \& Edelson 2001; Markowitz. Edelson \& Vaughan 2003). Summed spectra were constructed by combining the data from the individual $\sim 1 \mathrm{ks}$ observations within the defined time bins. A time-averaged model was applied to all binned spectra. The first five time bins were defined to match the start/stop times of the RXTE campaigns in 1997 March, 1999 January-February, 2001 March, 2001 September and 2003 September. For the sustained longterm monitoring that started on 2004 March 1, we considered all data acquired up to 2010 February 14. Bin sizes were chosen to optimize the trade-off between minimizing the uncertainties in the Fe line flux and maximizing the number of bins. This yielded 31 bins each of roughly 60 days in duration. The response of the
PCA and the conversion factor between count rate per PCU and incident flux, evolve slowly. In the $2-10 \mathrm{keV}$ band, $1.0 \mathrm{ct} / \mathrm{s} / \mathrm{PCU}$ corresponds to roughly $1.12 \times 10^{-11}$, $1.09 \times 10^{-11}$, and $1.04 \times 10^{-11} \mathrm{erg} \mathrm{cm}^{-2} \mathrm{~s}^{-1}$ during 1997 , 2003, and 2008, respectively. Consequently, regarding long-term PCA light curves, fluxes obtained from spectral fitting are more valuable than count rates. We have taken this long-term trend in response into account in our analysis. The number of PCUs used during the analysis of data from various epochs are as follows: PCU 0 , 1 and 2 for 1997 March, PCU 0 and 2 for 1999 Jan-Feb, since PCU 1 had started to have thermal breakdown in 1998, and PCU 2 only for all data from 2001 to 2010, since PCU 0 lost its propane layer in 2000. The number of counts in a typical binned spectrum ranged from $\sim 10^{5}$ to $10^{6}$.

We summed the PCA spectra from the individual observations within each time interval to create the binned spectra. PCA response matrices were generated for each individual observation using PCARMF v.11.7 and summed, weighted by exposure time, to yield a response for each binned spectrum. We did not include HEXTE data in the time-resolved fits, as those data yielded no additional model constraints compared to the PCA alone on these time scales given the flux level of 3C 111. Spectra were fit over the 3-25 keV energy range in XSPEC v.12.5.1k using a model of the same form as the best-fit to the long-term time-averaged spectrum determined by Rivers, Markowitz \& Rothschild (2011). Those authors performed joint RXTE PCA + HEXTE spectral fitting over the 3-100 keV energy band to a summed spectrum comprising all RXTE data on 3C 111 observed from 1997 March until 2008 July. The model we use thus consists of a power law with a photon index $\Gamma_{\mathrm{X}}$ allowed to vary after being initially set at 1.67 , plus a Gaussian component to model the Fe K $\alpha$ emission line, with photo-electric absorption in our Galaxy similar to that given above. The intensity of the Gaussian component $I_{\mathrm{FeK} \alpha}$ was allowed to vary; the energy centroid and width $\sigma$ were kept fixed at $6.19 \mathrm{keV}$ (rest-frame) and $0.72 \mathrm{keV}$, respectively, since there was no significant evidence to allow these parameters to vary from these best-fit time-averaged values. Rivers, Markowitz \& Rothschild (2011) found no significant evidence to warrant inclusion of a Compton hump in the model. Background level corrections were applied using RECORN within XSPEC.

Figure 2 shows the resulting light curves for the 2-10 $\mathrm{keV}$ continuum flux $F_{2-10}, \Gamma_{\mathrm{X}}, I_{\mathrm{FeK} \alpha}$, and the Fe line equivalent width $E Q W$. These data are given in Table 4 of the electronic version. Errors on $I_{\mathrm{FeK} \alpha}, \Gamma_{\mathrm{X}}$, and $E Q W$ were derived using the point-to-point variance 11 . We determined errors on values of $F_{2-10}$ within a time bin from the $2-10 \mathrm{keV}$ continuum light curve, using the mean flux error on the $\sim 1$ ks exposures in that time bin.

\subsection{Optical Monitoring}

We monitored 3C 111 in the optical $R$ band over a portion of the time span of the X-ray observations. The majority of the measurements in $R$ band are from the $2 \mathrm{~m}$ Liverpool Telescope (LT) at La Palma, Canary Is-

11 See $\S 3.3$ of Vaughan \& Edelson (2001) or $\S 3.3$ of Markowitz et al. (2003) for further details and the definition of the point-to-point variance. 
Table 2

X-ray (2.4-10 KeV; RXTE-PCA) light curve of 3C 111 from 2004 to 2010 (first 5 rows).

\begin{tabular}{cccccccc}
\hline \hline RJD $^{\mathrm{a}}$ & exp (s) & flux $^{\mathrm{b}}$ & err & $\alpha^{\mathrm{c}}$ & err & counts/s/PCU & err \\
\hline 3065.9070 & 2080. & $2.565 \mathrm{E}-11$ & $5.683 \mathrm{E}-13$ & -0.691 & 0.062 & $2.613 \mathrm{E}+00$ & $5.307 \mathrm{E}-02$ \\
3068.8816 & 752. & $1.975 \mathrm{E}-11$ & $8.709 \mathrm{E}-13$ & -0.530 & 0.123 & $2.035 \mathrm{E}+00$ & $8.308 \mathrm{E}-02$ \\
3072.8645 & 1952. & $1.503 \mathrm{E}-11$ & $5.269 \mathrm{E}-13$ & -0.672 & 0.096 & $1.544 \mathrm{E}+00$ & $4.893 \mathrm{E}-02$ \\
3075.8169 & 2032. & $1.458 \mathrm{E}-11$ & $5.173 \mathrm{E}-13$ & -0.657 & 0.100 & $1.480 \mathrm{E}+00$ & $4.770 \mathrm{E}-02$ \\
3080.0244 & 1936. & $1.292 \mathrm{E}-11$ & $5.291 \mathrm{E}-13$ & -0.394 & 0.112 & $1.329 \mathrm{E}+00$ & $4.920 \mathrm{E}-02$ \\
$\ldots$ & $\ldots$ & $\ldots$ & $\ldots$ & $\cdots$ & $\cdots$ & $\ldots$ & $\cdots$ \\
\hline
\end{tabular}

${ }^{a}$ Relative Julian Date: JD-2450000.

b Unit: ergs $\mathrm{cm}^{-2} \mathrm{~s}^{-1}$.

${ }^{\mathrm{c}} \alpha$ : Energy Index.

Table 3

$\mathrm{X}$-Ray Longlook light curve of 3C 111 from $X M M-N e w t o n$ in $2.4-10$ $\mathrm{keV}$ band on $2009 \mathrm{Feb}$ 16-17 (first 5 rows).

\begin{tabular}{ccc}
\hline \hline RJD $^{\text {a }}$ & Counts/s & error \\
\hline 4878.50 & 4.26 & 0.12 \\
4878.51 & 4.06 & 0.11 \\
4878.52 & 3.91 & 0.12 \\
4878.53 & 3.98 & 0.13 \\
4878.55 & 3.98 & 0.13 \\
$\ldots$ & $\ldots$ & $\ldots$ \\
\hline a Relative & Julian & Date: \\
JD-2450000. & &
\end{tabular}

lands, Spain, supplemented by observations at the $1.8 \mathrm{~m}$ Perkins Telescope of Lowell Observatory, Flagstaff, Arizona. We used star A (Figure 3) located $\sim 50^{\prime \prime}$ west of 3C 111 as a comparison star, for which we determined an $R$-band magnitude $R=14.93 \pm 0.05$ based on differential photometry with standard stars from other fields of view. Data acquisition and analysis procedures were identical to those described by Chatteriee et al. (2009). We also monitored the $R$-band linear polarization of $3 \mathrm{C} 111$ from 2006 to 2009 with the PRISM camera on the Perkins telescope at Lowell Observatory and with the $2.2 \mathrm{~m}$ telescope at Calar Alto Observatory, Spain I2. Interstellar polarization (ISP) is significant in the field of $3 \mathrm{C} 111$. We used the polarization parameters of the comparison star, presumed to be intrinsically unpolarized, to correct the polarization of 3C 111 for ISP (see also Jorstad et al. 2007). We corrected the degree of linear polarization for statistical bias using the method of Wardle \& Kronberg (1974).

\subsection{Radio Monitoring}

We monitored the $14.5 \mathrm{GHz}$ flux of $3 \mathrm{C} 111$ with the 26 $\mathrm{m}$ antenna of the University of Michigan Radio Astronomy Observatory. Details of the calibration and analysis techniques are described in Aller et al. (1985). The flux scale is set by observations of Cassiopeia A (Baars et al. 1977). We have compiled a $37 \mathrm{GHz}$ light curve with data from the $13.7 \mathrm{~m}$ telescope at Metsähovi Radio Observatory, Finland. The flux density scale is based on observations of DR 21, with 3C 84 and 3C 274 used as secondary calibrators. A detailed description of the

12 Data acquired as part of the MAPCAT program: http://www.iaa.es/ iagudo/research/MAPCAT

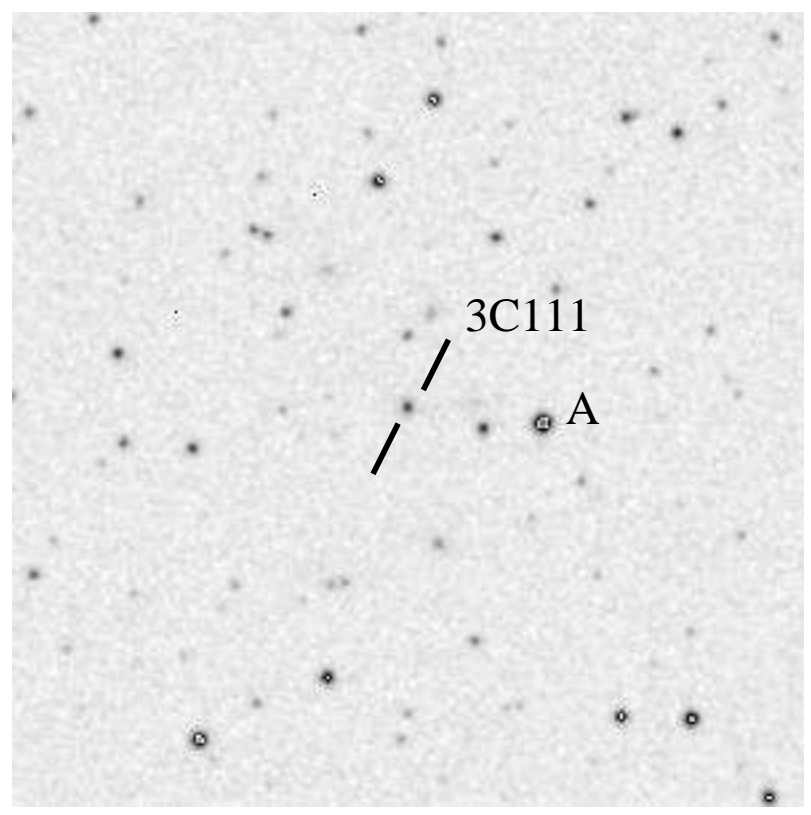

Figure 3. Finding chart for $3 \mathrm{C}$ 111. Star A is the comparison star with $R=14.93 \pm 0.05$, located $\sim 50^{\prime \prime}$ west of $3 \mathrm{C} 111$, used to calculate the magnitude and interstellar polarization correction $\left(p=3.6 \pm 0.2 \%, \chi=123^{\circ} \pm 2^{\circ}\right)$.

data reduction and analysis is given in Teräsranta et al. (1998). We have also compiled a $230 \mathrm{GHz}$ light curve of 3C 111 over a portion of the time span of the X-ray observations with calibration data from the the Submillimeter Array (SMA; see Gurwell et al. 2007, for details).

Figure 4 presents the X-ray, optical, and radio light curves. The variation of the total R-band flux, polarization percentage, and electric vector position angle (EVPA) are shown in Fig. 5. These data are given in Tables 5, 6, 7, 8, and 9] of the electronic version.

\subsection{VLBA Imaging}

Starting in 2004 May, we observed 3C 111 with the Very Long Baseline Array (VLBA) at $43 \mathrm{GHz}$ at roughly monthly intervals, with some gaps of 2-4 months. The sequence of images (Figure 6) from these data provide a dynamic view of the jet at an angular resolution $\sim 0.1$ milliarcseconds (mas) in the direction of the jet, corresponding to $0.094 \mathrm{pc}$ for an adopted Hubble constant of $H_{0}=70 \mathrm{~km} \mathrm{~s}^{-1} \mathrm{Mpc}^{-1}$. We processed the data in the same manner as described in Jorstad et al. (2005). We modeled the brightness distribution at each epoch with multiple components with circular Gaussian brightness 
Table 4

Variation of $2.4-10 \mathrm{keV}$ continuum flux, intensity of the Fe line, photon index, and the Fe line equivalent width derived from time-resolved spectral fits to the RXTE-PCA monitoring data shown in Figure 2

\begin{tabular}{|c|c|c|c|c|c|c|c|c|}
\hline $\mathrm{Yr}$ & $\mathrm{f}_{\text {cont }}{ }^{\mathrm{a}}$ & err & $\mathrm{f}_{\text {line }} \mathrm{b}$ & err & $\Gamma^{\mathrm{c}}$ & err & $\mathrm{EQW}^{\mathrm{d}}$ & err \\
\hline 1997.22 & 4.26 & 0.03 & 6.05 & 2.39 & 1.71 & 0.06 & 104.68 & 41.40 \\
\hline 1999.05 & 2.25 & 0.09 & 2.12 & 2.39 & 1.59 & 0.06 & 67.64 & 76.44 \\
\hline 2001.20 & 7.47 & 0.07 & 9.14 & 2.39 & 1.77 & 0.06 & 90.74 & 23.76 \\
\hline 2002.69 & 5.00 & 0.04 & 7.24 & 2.39 & 1.72 & 0.06 & 106.76 & 35.29 \\
\hline 2003.67 & 5.11 & 0.03 & 10.13 & 2.39 & 1.77 & 0.06 & 148.30 & 35.03 \\
\hline$\ldots$ & $\ldots$ & $\ldots$ & $\ldots$ & $\cdots$ & $\ldots$ & $\ldots$ & $\ldots$ & $\cdots$ \\
\hline $\begin{array}{l}2-10 \mathrm{keV} \\
\text { ontensity } \\
\text { photon } \\
\text { Fe line e }\end{array}$ & 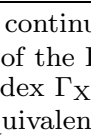 & $n \in$ & $\begin{array}{l}F_{2-1} \\
\text { eKa } \\
\text { ontin } \\
Q W\end{array}$ & $\begin{array}{l}10^{-} \\
-5 \mathrm{p} \\
\\
\text { V). }\end{array}$ & 8 & $\begin{array}{l}-2 \\
-1) .\end{array}$ & . & \\
\hline
\end{tabular}

Table 5

Optical (R Band) light curve of 3C 111 from 2004 to 2010 (first 5 rows).

\begin{tabular}{ccccc}
\hline \hline RJD $^{\mathrm{a}}$ & R Mag & err & Flux Density $^{\mathrm{b}}$ & err \\
\hline 3325.5154 & 17.033 & 0.009 & 0.474 & 0.004 \\
3325.5171 & 17.090 & 0.009 & 0.450 & 0.004 \\
3325.5210 & 17.047 & 0.009 & 0.468 & 0.004 \\
3325.5247 & 17.055 & 0.009 & 0.465 & 0.004 \\
3325.5266 & 17.030 & 0.009 & 0.475 & 0.004 \\
$\ldots$ & $\ldots$ & $\ldots$ & $\ldots$ & $\ldots$ \\
\hline
\end{tabular}

${ }^{a}$ Relative Julian Date: JD-2450000.

b Unit: mJy.

Table 6

Radio (14.5 GHz) light curve of 3C 111 from 2004 to 2010 (first 5 rows).

\begin{tabular}{ccc}
\hline \hline RJD $^{\text {a }}$ & Flux Density $(J y)$ & error \\
\hline 3024.5 & 2.97 & 0.10 \\
3042.5 & 3.06 & 0.04 \\
3050.5 & 3.16 & 0.04 \\
3052.5 & 3.09 & 0.04 \\
3064.5 & 3.02 & 0.03 \\
$\ldots$ & $\ldots$ & $\cdots$ \\
\hline a & $\cdots$ & $\ldots$
\end{tabular}

Table 7

Radio (37 GHz) light curve of 3C 111 from 2005 to 2008 (first 5 rows).

\begin{tabular}{ccc}
\hline \hline RJD $^{\text {a }}$ & Flux Density $(J y)$ & error \\
\hline 3371.22 & 4.61 & 0.25 \\
3372.20 & 4.35 & 0.24 \\
3379.52 & 4.19 & 0.22 \\
3423.03 & 4.23 & 0.23 \\
3447.93 & 4.17 & 0.26 \\
$\ldots$ & $\ldots$ & $\ldots$ \\
\hline a & $\ldots$ & $\ldots$
\end{tabular}

distributions using the task MODELFIT of the software package Difmap (Shepherd et al. 1997). This represents the jet emission downstream of the core, the bright feature situated at the western end of the jet, by a sequence
Table 8

Radio (230 GHz) light curve of 3C 111 from 2004 to 2010 (first 5 rows).

\begin{tabular}{ccc}
\hline \hline RJD $^{\mathrm{a}}$ & Flux Density $(\mathrm{Jy})$ & error \\
\hline 3022.48 & 1.26 & 0.09 \\
3031.60 & 1.71 & 0.35 \\
3266.67 & 2.06 & 0.13 \\
3359.38 & 2.49 & 0.13 \\
3381.64 & 3.80 & 0.20 \\
$\ldots$ & $\cdots$ & $\cdots$ \\
\hline
\end{tabular}

${ }^{a}$ Relative Julian Date: JD-2450000.

Table 9

Variation of the R-band Polarization Percentage and EVPA of 3C 111 from 2006 to 2010 .

\begin{tabular}{ccccc}
\hline \hline RJD $^{\mathrm{a}}$ & $\mathrm{p}(\%)^{\mathrm{b}}$ & error & EVPA $^{\mathrm{c}}$ & error \\
\hline 3741.74 & 3.32 & 0.60 & 42.70 & 5.20 \\
3796.65 & 0.60 & 0.80 & 97.50 & 38.30 \\
4048.87 & 3.33 & 0.70 & 65.80 & 6.00 \\
4049.98 & 3.06 & 0.40 & 22.70 & 3.70 \\
4076.88 & 1.49 & 0.80 & 26.00 & 15.40 \\
$\ldots$ & $\ldots$ & $\ldots$ & $\ldots$ & $\ldots$ \\
\hline
\end{tabular}

a Relative Julian Date: JD-2450000.

b R-band polarization percentage.

c Electric vector position angle.

of knots (also referred to as "components"), each characterized by its flux density, FWHM diameter, and position relative to the core. The ejection time $T_{0}$ is the extrapolated time of coincidence of a moving knot with the position of the (presumed stationary) core. Figure 7 plots the distance vs. epoch for all moving components brighter than 100 mJy within 2.0 mas of the core. We use the position vs. time data to determine the projected direction on the sky of the inner jet, as well as the apparent speeds and ejection times of new superluminal knots. Table 10 lists the values of $T_{0}$ and apparent speeds of new knots determined by the above procedure. In the top panel of Figure 4, the arrows represent the times of superluminal ejections, while the line segments perpendicular to the arrows show the uncertainties in the values of $T_{0}$. The apparent speeds of the components with well-determined motions are between 2.2 and $6.4 c$. 


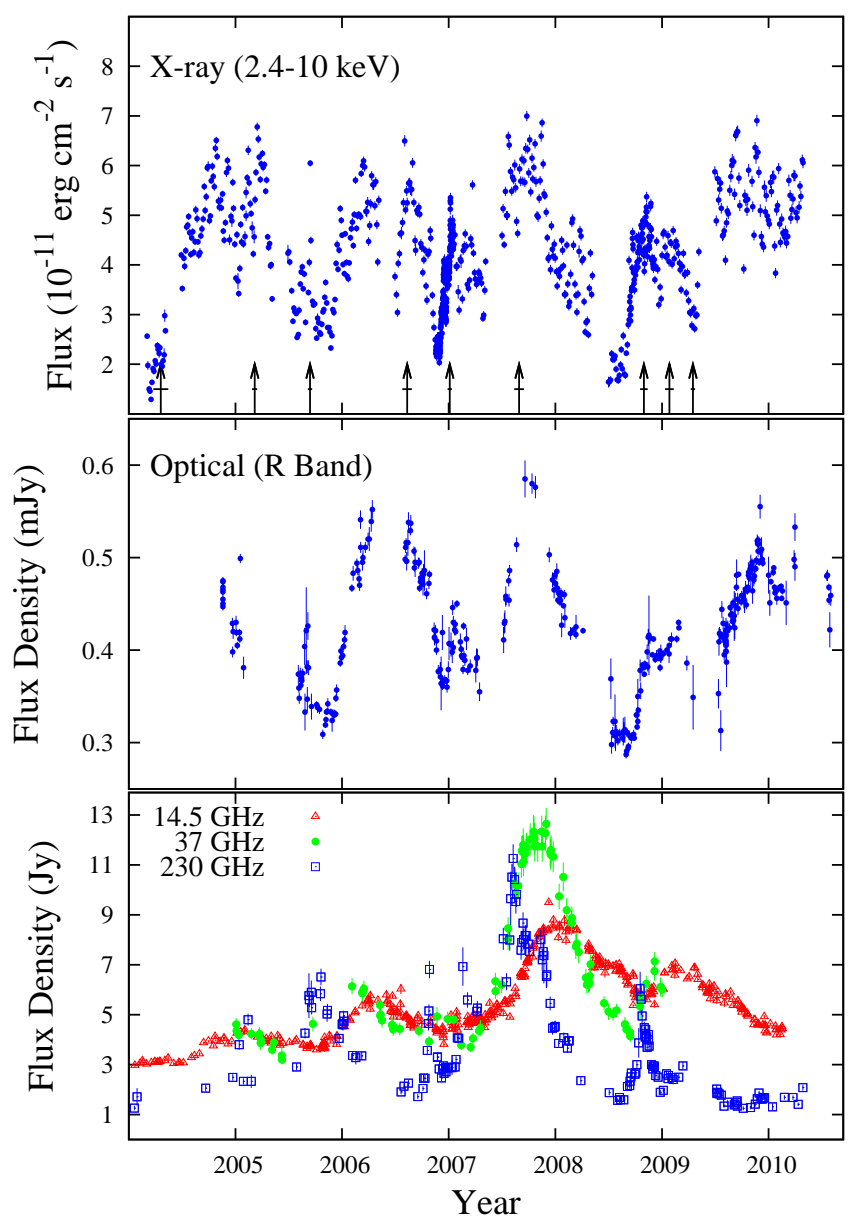

Figure 4. Variation of X-ray flux, optical flux density, and radio flux density of 3C 111 from 2004 to 2010 . In the top panel, the arrows indicate the times of superluminal ejections and the line segments perpendicular to the arrows represent the uncertainties in the times.

Table 10

Times of X-ray Dips, Ejection Times $\left(T_{0}\right)$ of Radio Knots, and Apparent Speeds $\left(\beta_{\mathrm{app}}\right)$ of Radio Knots.

\begin{tabular}{ccccc}
\hline \hline Dip ID & T Xmin ${ }^{\mathrm{a}}$ & Knot ID & $\mathrm{T}_{0}$ & $\beta_{\text {app }}$ \\
\hline $\mathrm{d} 1$ & 2004.19 & $\mathrm{~K} 1$ & $2004.30 \pm 0.14$ & $3.99 \pm 0.19$ \\
$\mathrm{~d} 2$ & 2005.02 & $\mathrm{~K} 2$ & $2005.18 \pm 0.05$ & $6.37 \pm 0.29$ \\
$\mathrm{~d} 3$ & 2005.57 & $\mathrm{~K} 3$ & $2005.70 \pm 0.04$ & $3.44 \pm 0.08$ \\
$\mathrm{~d} 4$ & 2006.47 & $\mathrm{~K} 4$ & $2006.61 \pm 0.09$ & $2.24 \pm 0.44$ \\
$\mathrm{~d} 5$ & 2006.88 & $\mathrm{~K} 5$ & $2007.01 \pm 0.04$ & $2.71 \pm 0.38$ \\
$\mathrm{~d} 6$ & 2007.32 & $\mathrm{~K} 6$ & $2007.66 \pm 0.09$ & $4.18 \pm 0.11$ \\
$\mathrm{~d} 7$ & 2008.51 & $\mathrm{~K} 7$ & $2008.83 \pm 0.07$ & $4.54 \pm 0.38$ \\
$\mathrm{~d} 8$ & 2008.98 & $\mathrm{~K} 8$ & $2009.07 \pm 0.08$ & $4.07 \pm 0.43$ \\
$\mathrm{~d} 9$ & 2009.26 & $\mathrm{~K} 9$ & $2009.29 \pm 0.04$ & $4.33 \pm 0.66$ \\
\hline
\end{tabular}

a Time of X-ray minimum.

\section{CORRELATED VARIATIONS IN THE X-RAY CONTINUUM AND FE K $\alpha$ EMISSION LINE}

Figure 8 shows the zero-lag correlation diagrams of $I_{\mathrm{FeK} \alpha}, \Gamma_{\mathrm{X}}$, and $E Q W$, each plotted against $F_{2-10}$. Bestfit linear relations are plotted for each and are summarized in Table 11 along with the Pearson correlation coefficients. From this diagram, we can see that the range of $F_{2-10}$ is from $2 \times 10^{-11}$ to $8 \times 10^{-11}$ (factor of $\sim 4$ ), while that of $I_{\mathrm{FeK} \alpha}$ is from $4 \times 10^{-5}$ to $12 \times 10^{-5}$ (factor of $\sim 3$ ).
Hence, we conclude that at least $75 \%$ of the Fe line flux responds to X-ray continuum variations. We searched for lags between $F_{2-10}$ and $I_{\mathrm{FeK} \alpha}$ with the interpolated cross correlation function (ICCF; Gaskell \& Peterson 1987; White \& Peterson 1994), and the discrete crosscorrelation function (DCCF; Edelson \& Krolik 1988), with errors determined using the Flux-Randomization and Random Subset Selection (FR-RSS) technique proposed by Peterson et al. (1998). We omitted the first five points (1997-2003) from this analysis due to the large data gaps present. The ICCF peak correlation coefficient was 0.61 at a continuum-to-line delay $\tau=0$ days, with a $1 \sigma$ upper limit to the lag of $|\tau|=88$ days (Figure 9). This implies that the bulk of the line flux originates within $~ 90$ light-days of the X-ray continuum source if it lies near the plane of the accretion disk. Figure 8 also demonstrates that $\Gamma_{\mathrm{X}}$ increases with $F_{2-10}$, similar to the behavior seen in many Seyfert galaxies that lack a strong jet contribution to the X-ray spectrum (Papadakis et al. 2002; Shih, Iwasawa \& Fabian 2002).

The X-ray continuum/Fe line correlation presented here is perhaps the strongest such correlation observed so far (cf., for example, the continuum-line correlation observed in NGC 3227 by Markowitz et al. 2009, where $\sim 50 \%$ of the line flux responded to the continuum 


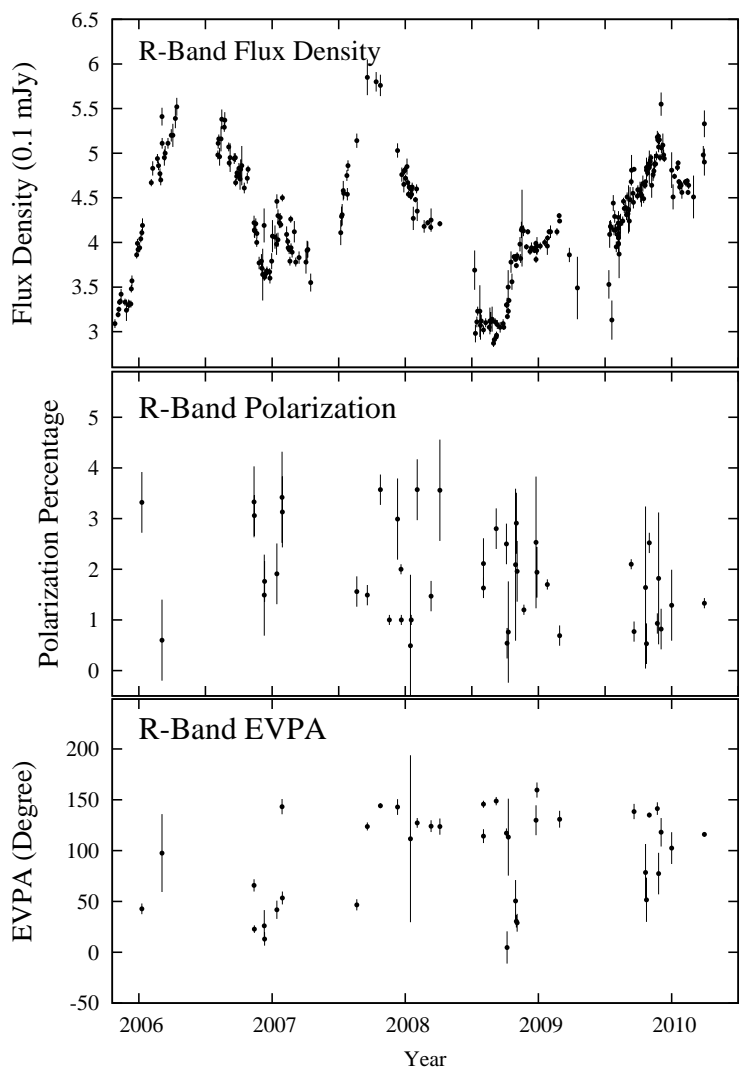

Figure 5. Variation of the optical R-band flux density, polarization percentage, and electric vector position angle (EVPA) of 3C 111 during 2006 to 2010. The average polarization was only $1.6 \% \pm 0.6 \%$, rather low for synchrotron radiation from the jet. This supports the notion that most of the optical emission is thermal radiation from the accretion disk.

Table 11

Best-fit Linear Relations For Parameters Derived From Time-resolved Spectroscopy.

\begin{tabular}{lcccc}
\hline \hline \multicolumn{1}{c}{ Parameters } & Slope & y-intercept & $r_{\mathrm{P}}$ & $P_{r}$ \\
\hline$I_{\text {FeK } \alpha}$ vs. $F_{2-10}$ & $1.27 \pm 0.31$ & $1.83 \pm 1.65$ & 0.594 & $1.3 \times 10^{-4}$ \\
$\Gamma_{\mathrm{X}}$ vs. $F_{2-10}$ & $0.040 \pm 0.008$ & $1.52 \pm 0.04$ & 0.796 & $5.8 \times 10^{-8}$ \\
$E Q W$ vs. $F_{2-10}$ & $-5.51 \pm 4.97$ & $150 \pm 29$ & -1.92 & 0.26 \\
\hline
\end{tabular}

Note. - The parameters for the best-fit linear solutions plotted in Figure 8 $r_{\mathrm{P}}$ is the Pearson correlation coefficient and $P_{r}$ is the null hypothesis probability, i.e., the probability of obtaining the observed correlation by chance.

variations on time scales of $\lesssim 700$ days). If the bulk of the $\mathrm{Fe}$ line originates within 90 light-days of the $\mathrm{X}$-ray continuum source, then an origin in material commensurate with the optical broad line region (located light-days to light-weeks away from the central black hole in Seyferts) is plausible. However, this delay is inconsistent with a model wherein the bulk of the line emission originates in a $\sim$ parsec-scale, homogeneous molecular torus commonly invoked in Seyfert $1 / 2$ unification schemes.

\section{POWER SPECTRAL ANALYSIS}

$$
\text { 4.1. X-ray }
$$

We use a variant of the Power Spectrum Response method (PSRESP; Uttley, McHardy \& Papadakis 2002) to determine the intrinsic PSD of the X-ray light curve. Our realization of PSRESP is described in Chatterjee et al. (2008). PSRESP gives both the bestfit PSD model and a "success fraction" $F_{\text {succ }}$ (fraction of simulated light curves that successfully represent the observed light curve) that indicates the goodness of fit of the model.

At first we fit a simple power-law model to the X-ray PSD, but found that the value of $F_{\text {succ }}$ is unacceptably low (0.1). This implies that a simple power law is not the best model for this PSD. We therefore fit a bending power-law model (broken power law with a smooth break) to the X-ray PSD (see McHardy et al. 2004; Chatterjee et al. 2009),

$$
P(\nu)=A \nu^{\alpha_{L}}\left[1+\left(\frac{\nu}{\nu_{B}}\right)^{\left(\alpha_{L}-\alpha_{H}\right)}\right]^{-1} .
$$

Here, $A$ is a normalization constant, $\nu_{B}$ is the break frequency, and $\alpha_{H}$ and $\alpha_{L}$ are the slopes of the power laws above and below the break frequency, respectively. During the fitting, we varied the break frequency $\nu_{\mathrm{B}}$ from $10^{-9}$ to $10^{-5} \mathrm{~Hz}$ in steps of $10^{0.05}, \alpha_{\mathrm{H}}$ (slope of the power law above the break frequency) from -1.5 to -3.0 in steps of 0.1 , and $\alpha_{\mathrm{L}}$ (slope below the break frequency) from -1.0 to -1.5 in steps of 0.1 . These ranges include the values of $\alpha$ found in the light curves of BHXRBs, for which $\alpha_{\mathrm{L}} \approx-1$ and $\alpha_{\mathrm{H}}$ is between -2 and -3 (e.g., Remillard \& McClintock 2006). This procedure yields a much higher success fraction than the simple power-law model. Based on the model with the highest success fraction (0.85), we obtain a good fit with the parameters $\alpha_{\mathrm{L}}=-1.0 \pm 0.1, \alpha_{\mathrm{H}}=-2.5_{-0.5}^{+0.2}$, and $\log _{10}\left(\nu_{\mathrm{B}}\right)=-6.05_{-0.30}^{+0.25} \mathrm{~Hz}$, which is equivalent to a timescale $13_{-6}^{+12}$ days. Figure 10 presents this best-fit model and the corresponding PSD. As seen in the figure, the high frequency part of the PSD is dominated by Poisson noise. That is because i) this part of the PSD is generated from the longlook light curve, and fluxes in the longlook light curve have larger uncertainties owing to shorter exposure times than those in the other light curves and ii) due to the red noise nature of the PSD, the intrinsic power is smaller at the higher frequencies and hence the power generated by Poisson noise is relatively more important. The figure shows that when the estimated Poisson noise is added to the best-fit model PSD, it matches the observed PSD quite well.

The PSD break frequency in BHXRBs and Seyfert galaxies scales inversely with the mass of the black hole (Uttley, McHardy \& Papadakis 2002; McHardy et al. 2004, 2006; Edelson \& Nandra 1999; Markowitz et al. 2003). McHardy et al. (2006) showed that this holds over a range of $\mathrm{BH}$ masses from 10 to $10^{8} \mathrm{M}_{\odot}$, and that, for a given black hole mass, the break frequency increases with accretion rate $(\dot{m})$. The bolometric luminosity $\left(L_{\mathrm{bol}}\right)$ can serve as a proxy for $\dot{m}$. Since the BH mass in 3C 111 is not well-established, we need to derive its value from independent observations. To do this, we need to determine the optical luminosity of the central engine. 

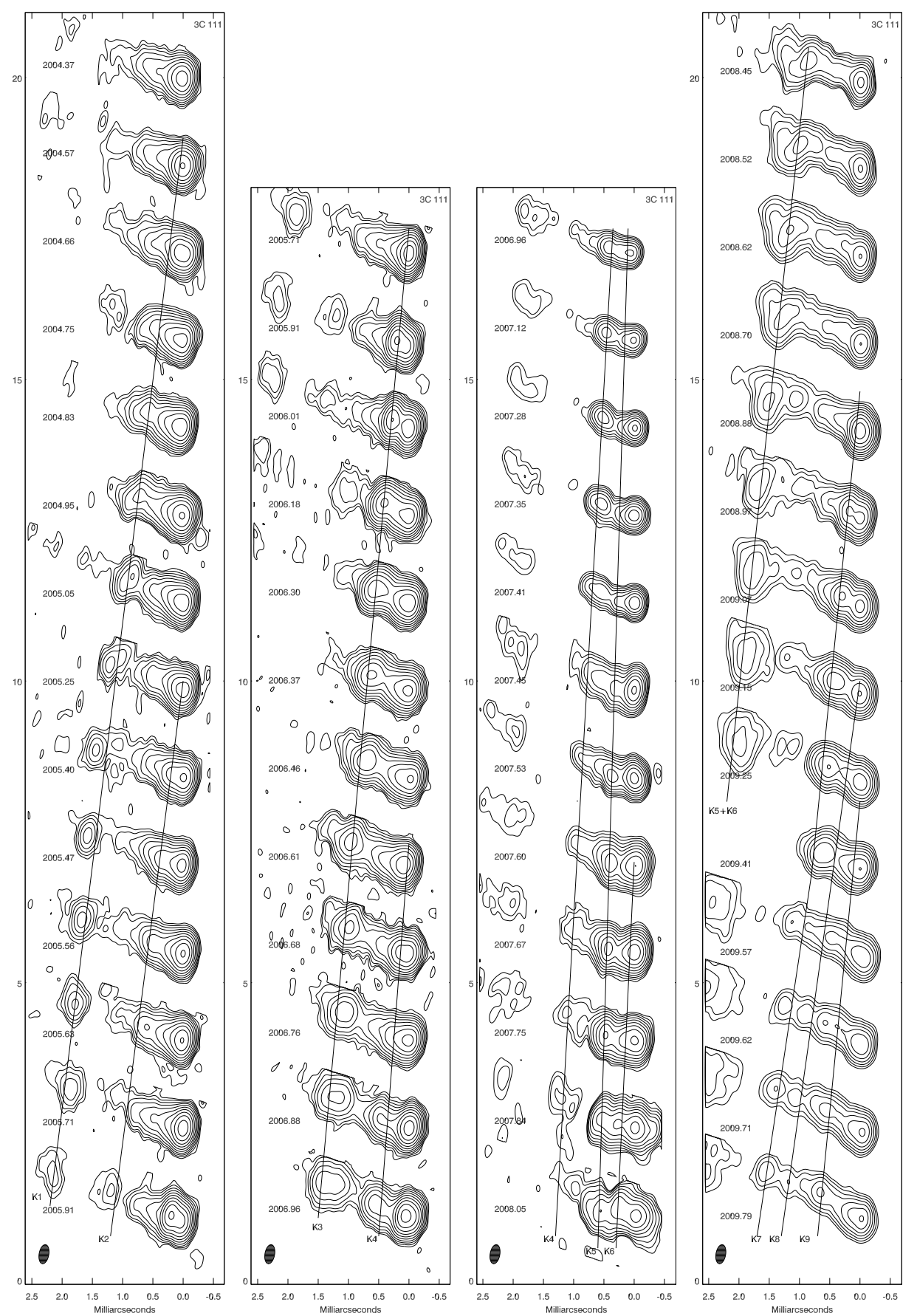

Figure 6. Sequences of VLBA images at $7 \mathrm{~mm}$ during 2004 to 2009. The images are convolved with an elliptical Gaussian beam of FWHM size $0.32 \times 0.16$ mas at PA $=-10^{\circ}$. The global peak over all maps is $4.80 \mathrm{Jy} /$ Beam. The contour levels are $0.25,0.354,0.5,0.707$, ..., $90.51 \%$ of the global peak. Note that the time spacing of the images is not uniform, hence the lines denoting the proper motion of a given knot do not pass through the centroid of the knot at every epoch.

The optical emission received from $3 \mathrm{C} 111$ is subject to substantial extinction owing to the presence of a translucent $\left(A_{\mathrm{v}}\right.$ between $\sim 1$ and 5$)$ molecular cloud in the foreground. The standard method for determining the extinction, star counts in the surrounding region on the sky, is subject to systematic uncertainties because of possible gradients in dust absorption. In fact, Marscher, Moore \& Bania (1993) and Moore \& Marscher (1995) have reported time variations in radio $\mathrm{H}_{2} \mathrm{CO}$ absorption lines toward $3 \mathrm{C} 111$, indicating that the column density and/or excitation conditions may vary throughout the cloud, causing changes in absorption as our line of sight to the quasar drifts across the cloud from relative motion between the Earth and the cloud. Sargent (1977) gives essentially simultaneous multi-band optical and near-infrared flux measurements of 3C 111, which are not corrected for extinction. We analyze this spectrum to calculate a value of the extinction, $A_{\mathrm{v}}=2.5$, that generates an extinction-corrected spectrum that can be fit well by a power law, the functional form that fits the optical continuum of a typical AGN. The spectral index of the extinction-corrected spectrum, 


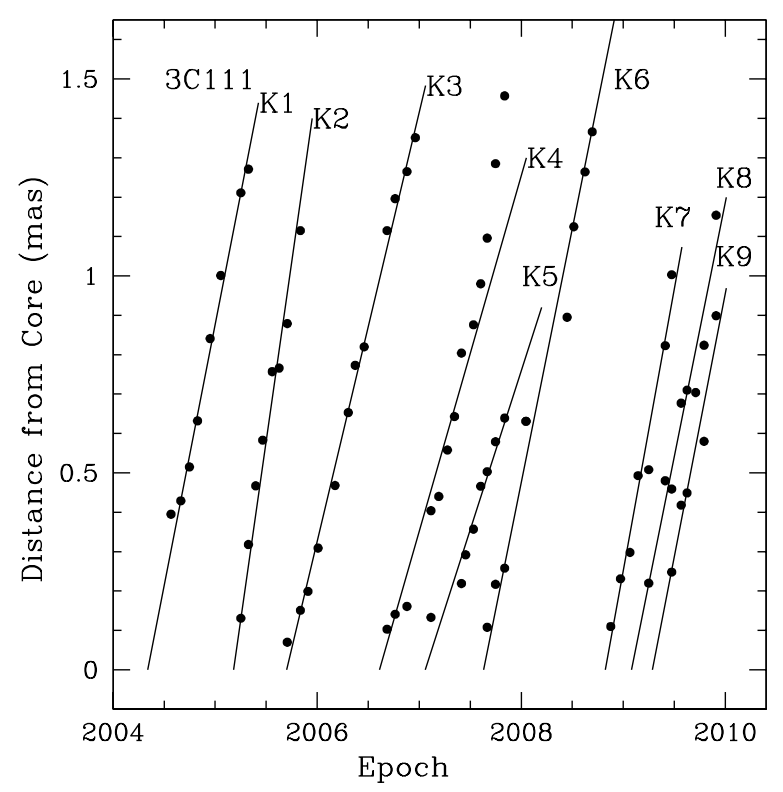

Figure 7. Angular separation from the core vs. epoch of the moving knots brighter than $100 \mathrm{mJy}$ within 2.0 mas of the core. The black lines indicate the motion of each knot listed in Table 10 A knot is identified through continuity of the trajectory from one epoch to the next. The identification of the knot across epochs considers its position angle relative to the core and, in some cases, its polarization. At some epochs, the model fit breaks a knot into two pieces.

displayed in Figure 11 is -1.38 . Our value of $A_{\mathrm{v}}$ is consistent with the values $\mathrm{E}(\mathrm{B}-\mathrm{V}) \sim 1$ for translucent clouds with total $\mathrm{N}(\mathrm{H}) \sim 9 \times 10^{21} \mathrm{~cm}^{-2}$ (Rachford et al. $2002)$ and $A_{\mathrm{v}} / \mathrm{E}(\mathrm{B}-\mathrm{V}) \sim 2.1$ derived for the translucent cloud HD 36982 by Larson. Whittet \& Hough (1996). We note, however, that these values have high uncertainties and could differ significantly from one cloud to another. After correcting the optical luminosity from Sargent (1977) by this value of $A_{\mathrm{v}}$, we arrive at a dereddened optical luminosity of $\lambda L_{\lambda}=6.1 \times 10^{43} \mathrm{erg} \mathrm{s}^{-1}$ at $\lambda=510 \mathrm{~nm}$.

In a recent paper, Decarli, Dotti \& Treves (2011) used the properties of the $\mathrm{H} \alpha$ line of a sample of blazars in addition to other parameters to determine the mass of the central $\mathrm{BH}$. Using the deprojection factor and $\mathrm{H} \alpha$ line to continuum $(510 \mathrm{~nm})$ luminosity ratio from equation (3) and (5), respectively, of that paper, as well as the width and luminosity of the $\mathrm{H} \alpha$ line from Eracleous \& Halpern (2003), we calculate the $\mathrm{BH}$ mass of $3 \mathrm{C} 111, M_{\mathrm{BH}}=$ $2.4_{-0.5}^{+0.6} \times 10^{8} \mathrm{M}_{\odot}$. Another method for estimating the $\mathrm{BH}$ mass is to use the relationship between $M_{\mathrm{BH}}$ and the FWHM line width of the $\mathrm{H} \beta$ broad emission line along with equation (2) of Vestergaard \& Peterson (2006). Unfortunately, no accurate measurement of $\operatorname{FWHM}(\mathrm{H} \beta)$ in $3 \mathrm{C} 111$ has been published; instead, we assume that $\operatorname{FWHM}(\mathrm{H} \beta) \approx \operatorname{FWHM}(\mathrm{H} \alpha)=4800 \mathrm{~km} \mathrm{~s}^{-1}$ (Eracleous \& Halpern 2003). From this value and the de-reddened optical luminosity given above, we derive $M_{\mathrm{BH}}=1.5_{-0.3}^{+0.4} \times 10^{8} \mathrm{M}_{\odot}$. If we use the more conservative estimate of the uncertainty in the zero point value of the scaling relation between the masses determined using reverberation mapping and those from single-epoch spectra from Vestergaard \& Peterson (2006), then the respective

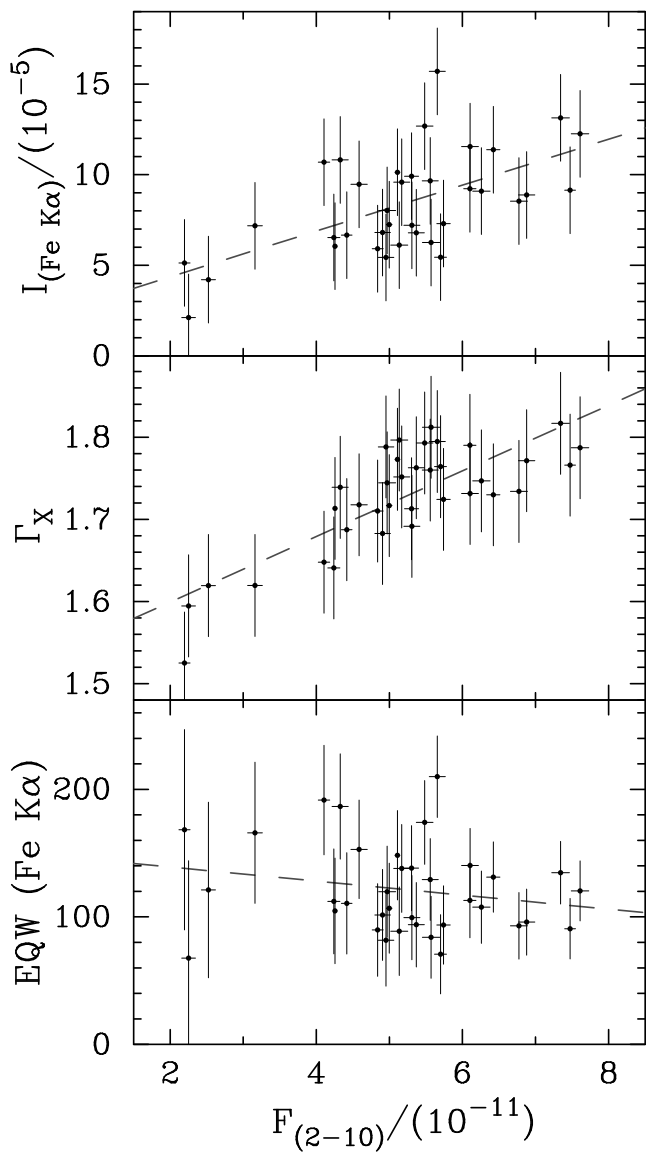

Figure 8. Zero-lag correlation diagrams for intensity of the Fe line $I_{\mathrm{FeK} \alpha}$, photon index $\Gamma_{\mathrm{X}}$ (of the continuum), and Fe line equivalent width $E Q W$ each plotted against $2-10 \mathrm{keV}$ continuum flux $F_{2-10}$ with the best-fit linear relation plotted for each as a dashed line; units are the same as in Figure 2 The X-ray continuum/Fe line correlation presented here is one of the strongest such correlation observed so far. The best-fit linear relations plotted here are summarized in Table 11 along with the Pearson correlation coefficients.

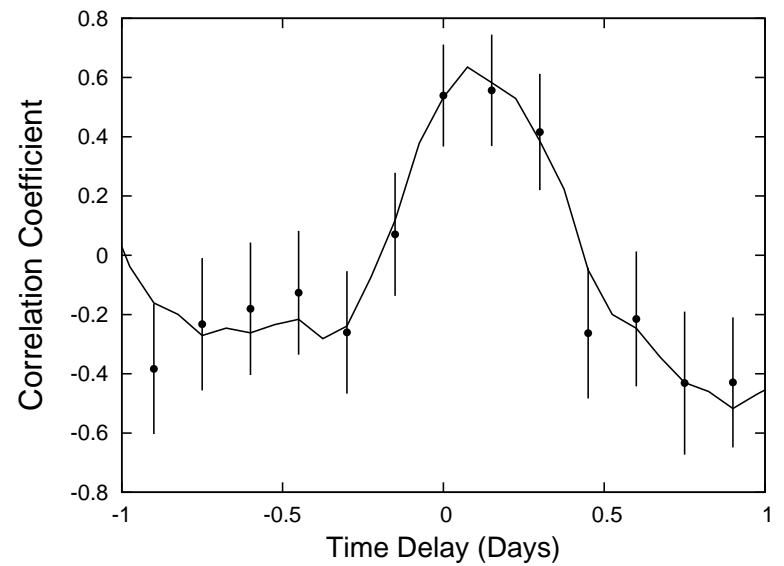

Figure 9. Points denote discrete cross-correlation function (DCCF), and the solid line shows the interpolated crosscorrelation function (ICCF) of the variation of intensity of the Fe line $I_{\mathrm{FeK} \alpha}$ with that of $2-10 \mathrm{keV}$ continuum flux $F_{2}-10$. The two variations are strongly correlated with a time delay consistent with zero with a $1 \sigma$ upper limit of 88 days. 


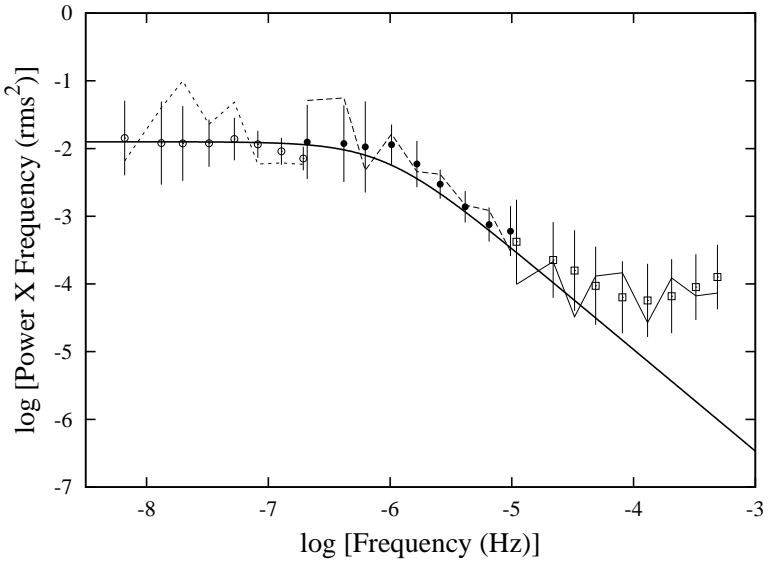

Figure 10. Result of application of the PSRESP method to the X-ray light curve of 3C 111. The PSD of the observed data at high, medium, and low frequencies is given by the solid, dashed, and dotted jagged lines, respectively, while the underlying power-law model is given by the thicker solid bent line. Points with error bars (open squares, filled circles, and open circles for high, medium, and low frequency range, respectively) correspond to the mean value of the PSD simulated from the underlying power-law model (see text). The error bars are the standard deviations of the distribution of simulated PSDs. The broadband power spectral density is best described by a bending power law with low frequency slope -1.0 , high frequency slope -2.5 , and break frequency $10^{-6.05} \mathrm{~Hz}$. The high frequency part of the PSD is dominated by Poisson noise. The figure shows that when the estimated Poisson noise is added to the underlying bending power-law model PSD, it matches the observed PSD quite well.

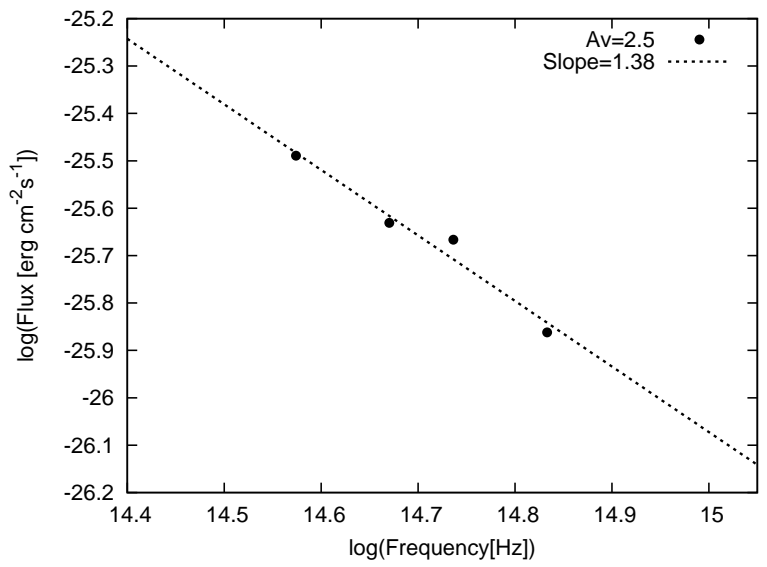

Figure 11. The extinction corrected optical spectrum of $3 \mathrm{C} 111$. The best-fit power-law has a slope -1.38 for $A_{\mathrm{v}}=2.5$.

uncertainty in the value of the $\mathrm{BH}$ mass becomes larger, $M_{\mathrm{BH}}=1.5_{-1.0}^{+2.5} \times 10^{8} \mathrm{M}_{\odot}$. Using the correlation between the widths of $\mathrm{H} \alpha$ and $\mathrm{H} \beta$ emission lines from equation (3) of Greene \& Ho (2005), we calculate $\operatorname{FWHM}(\mathrm{H} \beta)$ of $3 \mathrm{C} 111$ to be $5400 \pm 400 \mathrm{~km} / \mathrm{s}$ from $\operatorname{FWHM}(\mathrm{H} \alpha)=$ $4800 \mathrm{~km} \mathrm{~s}^{-1}$ (Eracleous \& Halpern 2003). Using this value in equation (2) of Vestergaard \& Peterson (2006) and keeping other parameters same as above, we obtain $M_{\mathrm{BH}}=1.8_{-0.4}^{+0.5} \times 10^{8} \mathrm{M}_{\odot}$.

Marchesini. Celotti \& Ferrarese (2004) derived a much higher value of $M_{\mathrm{BH}}$ estimating the bolometric luminosity of $3 \mathrm{C} 111$ from the optical nuclear luminosity along with the bolometric correction from Elvis et al. (1994). The former is derived by fitting the unresolved nuclear

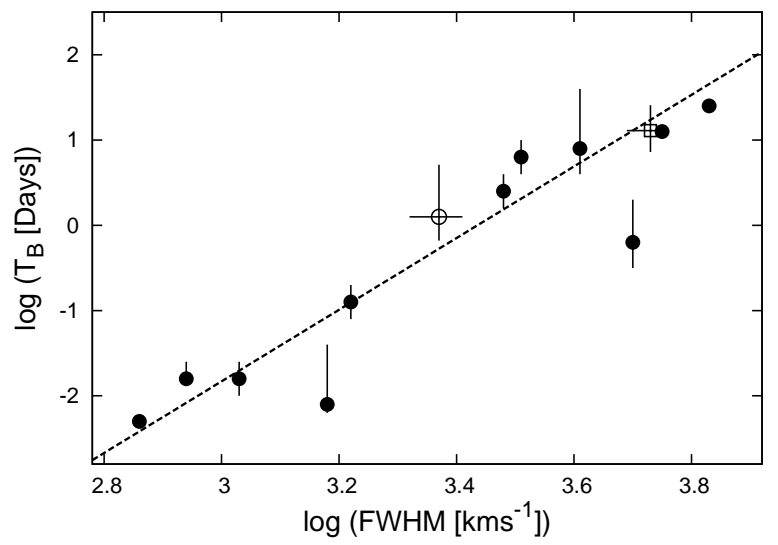

Figure 12. The correlation of $\mathrm{H} \beta$ line-width with the PSD break timescale for a sample of mainly radio-quiet AGNs from McHardy et al. (2006) is denoted by the dashed straight line and the solid circles. The open circle and the open square show data points corresponding to radio galaxies $3 \mathrm{C} 120$ and $3 \mathrm{C} 111$, respectively, which follow the correlation. For 3C 111, line width of $\mathrm{H} \beta$ has been calculated from that of $\mathrm{H} \alpha$ using equation (3) of Greene \& Ho (2005)

component with the appropriate HST PSF in the respective HST image. They adopted an extinction correction from Schlegel, Finkbeiner \& Davis (1998) which is higher than the extinction we calculated above by almost 3 magnitudes. If we adjust the calculation of Marchesini. Celotti \& Ferrarese (2004) to our extinction correction of $A_{\mathrm{v}}=2.5$, we obtain $L_{\mathrm{bol}}=(2.5 \pm 1.0) \times 10^{44}$ $\operatorname{ergs~s}^{-1}$. We also calculate $L_{\text {bol }}=(4.8 \pm 3.3) \times 10^{44}$ ergs s${ }^{-1}$ from Young. Elvis. \& Risaliti (2010) using the 2.4-10 keV X-ray flux and $510 \mathrm{~nm}$ optical flux, which is consistent with the above value within uncertainties. Performing a similar calculation using equation (11) of Lusso et al. (2010), we find $L_{\mathrm{bol}}=(1.8 \pm 1.1) \times 10^{45} \mathrm{ergs}$ $\mathrm{s}^{-1}$, which is close to the above values as well.

Using the intermediate values from above, $L_{\mathrm{bol}}=$ $(4.8 \pm 3.3) \times 10^{44} \mathrm{erg} / \mathrm{s}$, and $M_{\mathrm{BH}}=1.8_{-0.4}^{+0.5} \times 10^{8} \mathrm{M}_{\odot}$, we find Eddington ratio $L_{\mathrm{bol}} / L_{\text {edd }}=0.02 \pm 0.01$. We adopt the best-fit values and uncertainties in the $T_{B}$ $\mathrm{M}_{\mathrm{BH}}-\mathrm{L}_{\mathrm{bol}}$ relation proposed by McHardy et al. (2006), and the above values of $M_{\mathrm{BH}}$ and $L_{\mathrm{bol}}$. We then calculate the expected break frequency to be $10^{-6.6 \pm 0.7} \mathrm{~Hz}$. If we use the more conservative estimate of the uncertainty in $\mathrm{BH}$ mass from above, we obtain $10^{-6.6 \pm 1.1} \mathrm{~Hz}$ for the same. Both of these are consistent with the value we have obtained from the best-fit PSD, $\log _{10}\left(\nu_{\mathrm{B}}\right)=-6.05_{-0.30}^{+0.25}$ $\mathrm{Hz}$, within uncertainties. This demonstrates that 3C 111 also follows the $\mathrm{T}_{\mathrm{B}}-\mathrm{M}_{\mathrm{BH}}-\mathrm{L}_{\mathrm{bol}}$ relation, the first FR II radio galaxy for which this has been tested.

McHardy et al. (2006) also showed that FWHM(H $\beta$ ) in AGNs is strongly correlated with the observed PSD break timescale $\left(T_{B}\right)$ given by

$$
\log \left(T_{B}\right)=4.2 \log ([F W H M(H \beta)])-14.43
$$

Using the results of the above analysis and that contained in Chatteriee et al. (2009), we can now add data points corresponding to the radio galaxies 3C 120 and 3C 111 in Figure 3 of McHardy et al. (2006), resulting in Figure 12. The plot shows that these two radio galaxies also follow the empirical $T_{B}$ versus $\operatorname{FWHM}(\mathrm{H} \beta)$ correlation. 


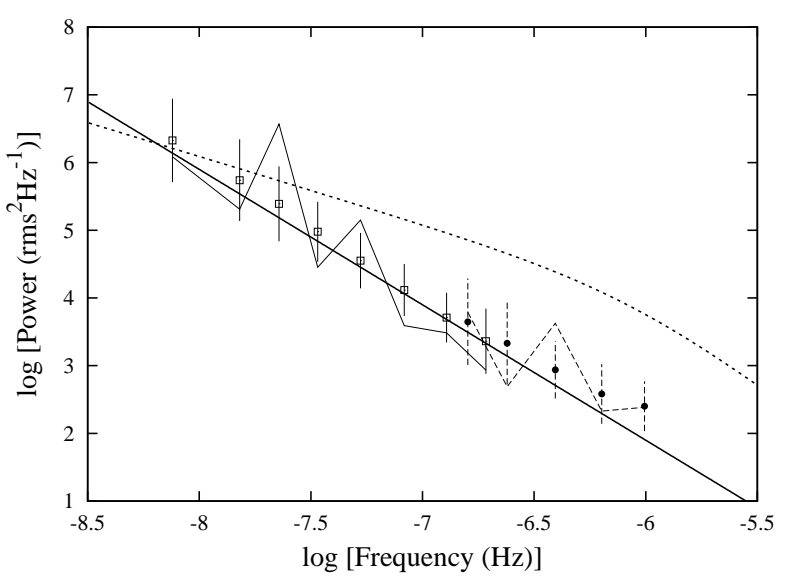

Figure 13. Result of application of the PSRESP method to the optical light curve of 3C 111. The PSD of the observed data at high and medium frequencies is given by the dashed and solid jagged lines, respectively, while the underlying power-law model is given by the solid straight line. Points with error bars (filled circles and open squares for high and medium frequency range, respectively) correspond to the mean value of the PSD simulated from the underlying power-law model (see text). The error bars are the standard deviations of the distribution of simulated PSDs. The broadband power spectral density is best described by a simple power law with slope -2.0 . The smoothly bending dotted line is the underlying best-fit bending power-law model of the X-ray PSD from Fig. 10 This shows that the X-ray variability has more power than that in the optical on shorter timescales while on longer timescales variability at the two wave bands tend to be similar.

\subsection{Optical}

We use the same method as above to calculate the PSD of the optical variability. The optical $R$-band PSD of 3C 111 (Figure 13) shows red noise behavior, i.e., there is higher amplitude variability on longer than on shorter timescales. Based on the model with the highest success fraction, the optical PSD is best fit with a simple power law of slope $-2.0_{-0.7}^{+0.3}$, for which the success fraction is 0.52. During the fitting, we varied the slope from -1.0 to -3.0 in steps of 0.1 . The rejection confidence, equal to one minus the success fraction, is much less than 0.9 , hence the model provides an acceptable fit to the PSD. We also fit a broken power law to the optical PSD, setting the low-frequency slope at -1.0 and allowing the break frequency and the slope above the break to vary over a wide range of parameters $\left(10^{-8}\right.$ to $10^{-6} \mathrm{~Hz}$ and -1.0 to -2.5 , respectively) while calculating the success fractions. This gives lower success fractions than the simple power-law model across the entire parameter space. Since we do not have any significantly long segment of the $R$-band data when the sampling frequency was more than 2-3 days per week, we determine the optical PSD up to the highest variational frequency that can be achieved with the existing data. A better constraint on the existence of a break in the optical PSD could be achieved with a broader range of sampled frequencies.

The slope of the optical PSD in the range $10^{-8.1}$ to $10^{-5.8} \mathrm{~Hz}$ is significantly steeper than that in X-rays over the same range $(\sim 1.0)$. Hence, the ratio of shorter to longer timescale variability amplitude is smaller at optical than at X-ray wavelengths.

\subsection{Excess Variance}

We calculate the fractional root mean squared variability amplitude, i.e., the "excess variance" normalized by the square of the mean flux, as a measure of the variability in the X-ray and optical data. It is defined as

$$
F_{\mathrm{var}}=\sqrt{\frac{\mathrm{S}^{2}-\overline{\sigma_{\mathrm{err}}^{2}}}{\bar{x}^{2}}},
$$

where $\mathrm{S}$ is the variance, $\sigma_{\text {err }}$ is the observational uncertainty, and $\bar{x}$ is the mean of the data (Nandra et al. 1997; Edelson et al. 2002; Vaughan et al. 2003). We find that the values of $F_{\text {var }}$ of the X-ray and optical variability for the entire data set are 0.28 and 0.14 , respectively. This supports the above conclusion from the PSD analysis that the optical is less strongly variable than the X-ray flux at the longest timescales we probe. It also implies that optical variations cannot be completely responsible for driving all of the X-ray variability observed, if we assume that most of the X-ray emission we observe is not relativistically beamed.

\section{X-RAY/RADIO CORRELATION}

We can see in Fig. 4 that, from the middle of 2009 to the present, the X-ray flux undergoes only minor fluctuations about a mean value of $5 \times 10^{-11} \mathrm{erg} \mathrm{cm}^{-2} \mathrm{~s}^{-1}$. During this interval, the $230 \mathrm{GHz}$ flux is at a very low level, while the $15 \mathrm{GHz}$ flux is decreasing. Therefore, the high-state X-ray plateau coincides with a low state of the radio jet. We can extend this $\mathrm{X}$-ray/radio connection to the observations of earlier years as well. The times of "ejection" of new radio knots in the jet (shown by the arrows) are related to the low X-ray states. We convolve the X-ray light curve with a Gaussian smoothing function with a 10-day FWHM smoothing time to identify the major long-term trends. Figure 14 shows the smoothed $\mathrm{X}$-ray light curve and denotes the times of superluminal ejections with arrows. Based on inspection of this light curve, we consider an X-ray fluctuation to be a significant dip if the smoothed X-ray flux falls by more than $30 \%$ and remains at a low level for longer than one month. We calculate the central time of each dip by determining the local minimum of the X-ray flux. We note that the minimum amplitude measured from unsmoothed data of every dip is below the average of the X-ray flux over the length of the light curve $\left(4 \times 10^{-11} \mathrm{erg} \mathrm{cm}^{-2} \mathrm{sec}^{-1}\right)$. It can be seen that each of the ejections is preceded by a significant dip in the X-ray flux. The prolonged low level of X-ray flux in 2005 is associated with knot K3, which was extended along the jet axis, as is apparent in the images starting in late 2006 (Fig. 6). This suggests that a prolonged disturbance near the base of the jet resulted in an elongated superluminal knot. Hence, d3 and the two significant dips just before and after that are considered to be one dip with multiple branches and is related to K3. Detection of the ejection of a new knot requires the analysis of a sequence of VLBA images of sufficient duration to define the trajectory and speed of a knot, as is evident from Figures [6] and [7. No new bright, moving knots after K9 appeared in subsequent imaging with the VLBA during the first 8 months of 2010 (images not shown here).

The time delay between the minimum of the X-ray dips and the time of ejection of the corresponding superlumi- 


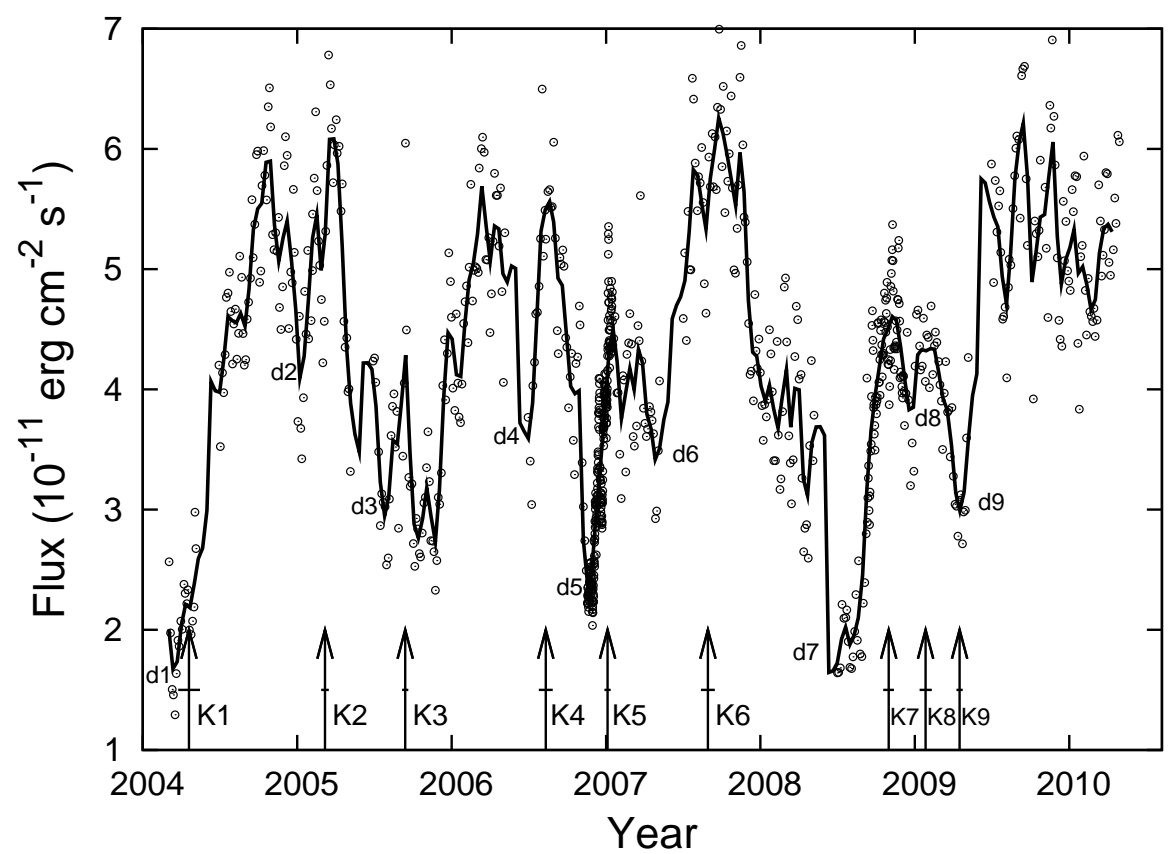

Figure 14. X-ray light curve of 3C 111 as in Figure 4 with data indicated by small circles. The curve corresponds to the same data, smoothed with a Gaussian function with a 10-day FWHM smoothing time. The arrows indicate the times of superluminal ejections and the line segments perpendicular to the arrows represent the uncertainties in the times. X-ray dips and VLBA knots listed in Table 10 are marked with the respective ID numbers. Over the six years of observation, significant dips in the X-ray light curve are followed by ejections of bright superluminal knots in the VLBA images. This shows a clear connection between the radiative state near the black hole, where the X-rays are produced, and events in the jet. Dip d3 and the two significant dips just before and after that are considered to be one dip with multiple branches and is related to K3 which was also extended along the jet axis.

nal knot is distributed between 0.03 and $0.34 \mathrm{yr}$ (Table 10) with a mean of $0.15 \pm 0.08 \mathrm{yr}$. We plot the times of ejection of new knots along with the corresponding times of X-ray dips in Figure [15] A straight line fits the data extremely well with small scatter, which indicates that there is a clear association between X-ray dips and superluminal ejections. The best-fit line through the points has a slope of 1 and y-intercept of 0.15 , which is identical to the mean delay of $0.15 \pm 0.08 \mathrm{yr}$ given above. We have performed a numerical simulation to calculate the significance of the dip-ejection correlation. If we keep the times of dips fixed and choose one million sets of nine ejections, times of which are drawn from a uniform random distribution between 2004.3 and 2010.3 (the duration of our observations), the probability that there is at least one ejection event following all 9 dips by 0.03 to $0.34 \mathrm{yr}$ is 0.007 . In a similar simulation, we found that the probability that all 9 dips will have exactly one following ejection is $3 \times 10^{-5}$. This shows that the dip-ejection association in 3C 111 is highly significant. To further investigate the relationship between the characteristics of the X-ray dips and VLBA knots, we calculate the average flux over all epochs of the latter. We do this via modeling of the brightness distribution at each epoch with multiple components characterized by circular Gaussian brightness distributions, as described in $\S 2.4$. This shows that the average flux of the VLBA knots corresponding to the shallower dips $(\mathrm{d} 2, \mathrm{~d} 4$, and $\mathrm{d} 8$ in Fig. (14) is significantly smaller $(0.3 \pm 0.1 \mathrm{Jy})$ than that for the larger dips $(\mathrm{d} 1, \mathrm{~d} 3, \mathrm{~d} 5$, and $\mathrm{d} 7), 0.9 \pm 0.4 \mathrm{Jy}$. Dip d6 is also shallow, but this may be due to the absence of data during the Sun-avoidance gap, while $\mathrm{d} 9$ is of intermediate depth. Hence K6 and K9 were not included in

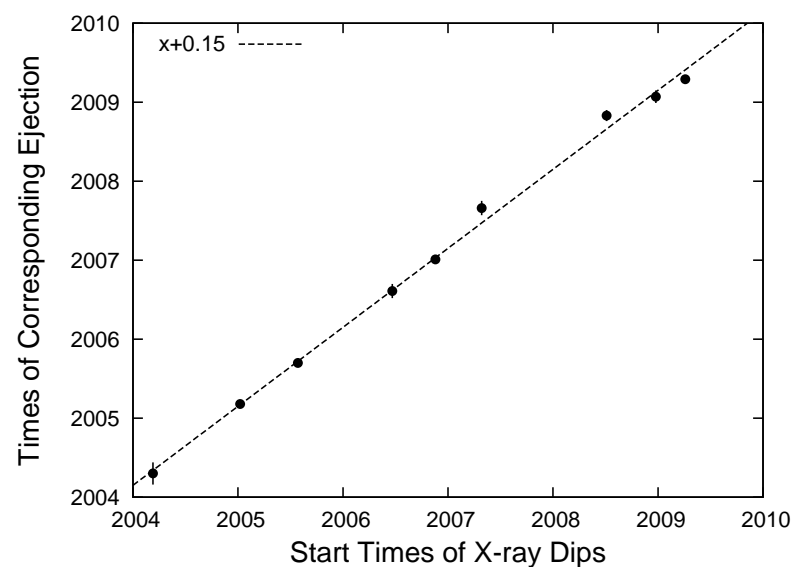

Figure 15. Times of ejection of new VLBA knots versus times of X-ray dips from Table 10 The dashed line is the best-fit straight line through the points. It fits the data extremely well with small scatter. This indicates that there is a clear association between $\mathrm{X}$-ray dips and superluminal ejections. The y-intercept of this line minus 2004.0 indicates the value of mean time delay between the times of dips and ejections.

the average calculation. That the brighter knots correspond to more pronounced dips supports the conclusion that the X-ray minima and the ejection of knots in the jet are physically related.

As determined by the discrete cross-correlation function (DCCF; Edelson \& Krolik 1988) shown in the top panel of Figure [16] the X-ray flux variations are weakly correlated with those at $15 \mathrm{GHz}$ in $3 \mathrm{C} 111$. The peak $\mathrm{X}$-ray versus $15 \mathrm{GHz}$ DCCF is 0.35 . We simulate Xray light curves from the underlying PSD as determined 


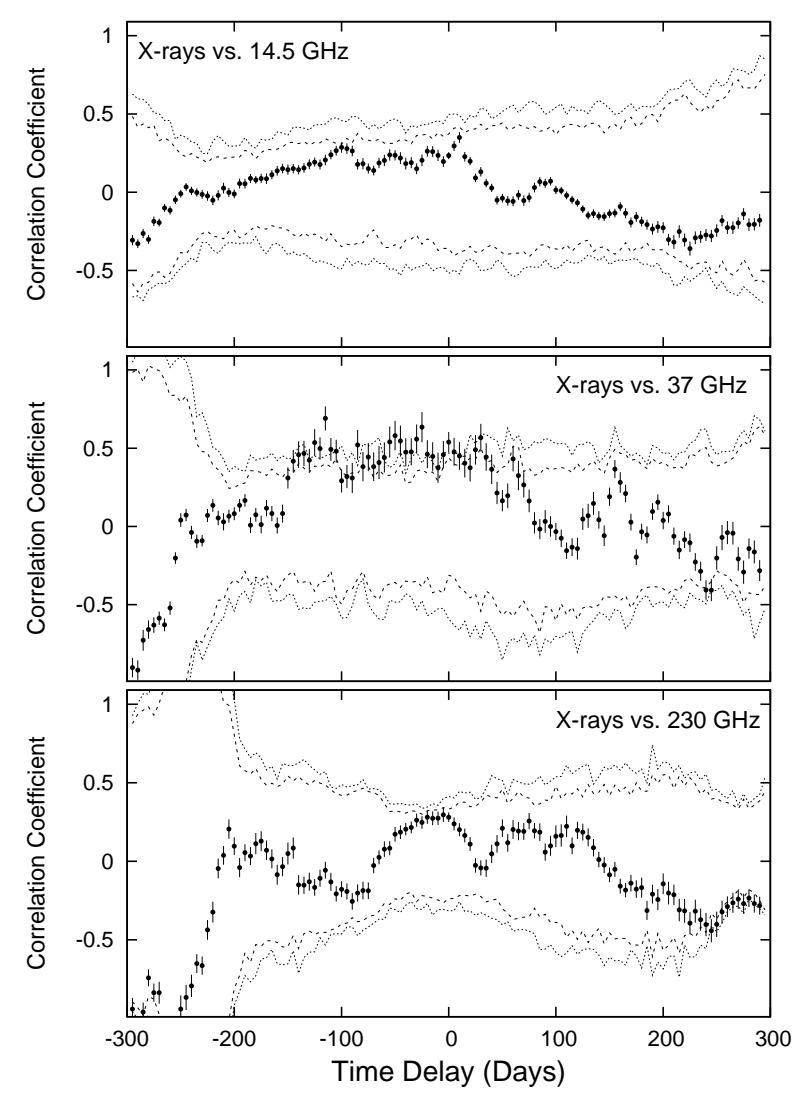

Figure 16. Discrete cross-correlation function (DCCF) of the $\mathrm{X}$-ray and radio monitoring data of $3 \mathrm{C} 111$. The time delay is defined as positive if the $\mathrm{X}$-ray variations lag those at radio frequencies. Top panel shows the correlation function for X-ray versus $15 \mathrm{GHz}$ variations while the middle and bottom panels show the same for X-ray versus 37 and $230 \mathrm{GHz}$, respectively. The jagged dotted and dashed lines at the top of each panel denote 99 and 95 percent significance levels of correlation, respectively (see text). Those at the bottom of each panel show the same for anticorrelation. This shows that the X-ray and $37 \mathrm{GHz}$ variations are correlated while the other two pairs are not significantly correlated.

in $\S 3$ and resample the simulated light curves with the sampling window of the actual X-ray light curve. Then we cross-correlate the resampled simulated X-ray light curves with the real $15 \mathrm{GHz}$ light curve. The solid and dotted jagged lines in Figure 16 indicate $99 \%$ and $95 \%$ extremes of the distribution of correlation coefficients as a function of time delay.

The DCCF indicates that the X-ray flux variations are correlated with those at $37 \mathrm{GHz}$ in $3 \mathrm{C} 111$, with a peak value of 0.63 (middle panel of Figure 16). The position of the peak of the correlation function indicates the relative time delay between the variations at the two wavelengths. The time lag of the peak corresponds to X-ray variations leading those at $37 \mathrm{GHz}$ by $40_{-65}^{+70}$ days. We use the FR-RSS technique (Peterson et al. 1998) to calculate the mean value and the uncertainty of the cross-correlation time lag. The bottom panel of Figure [16] shows that the X-ray and $230 \mathrm{GHz}$ variations are weakly correlated, with a peak DCCF of 0.30 .

We note that if the radio flux increases within tens of days after an X-ray minimum and the X-ray flux recovers from the minimum to increase as well during this time, then a relatively weak X-ray/radio correlation will result as seen in the data presented in this paper. The significant correlation between the dips and ejections of new knots in the jet implies that increased activity in the radio jet of $3 \mathrm{C} 111$ is related to a temporary decrease in $\mathrm{X}$-ray production. Similarly, the association between the high-state X-ray plateau with a low state of the radio jet after the middle of 2009 suggests that a persistently high $\mathrm{X}$-ray flux is associated with a low radio state.

It is possible that the "corona," where the X-ray emission seen in AGN is thought to arise from Compton upscattering of softer accretion-disk photons, might be the base of the jet (Markoff. Nowak, \& Wilms 2005). If this is the case, then the X-ray flux will be related to the number of electrons residing there and available for scattering to create X-rays. If the same number of electrons is injected into the base of the jet per unit time, then faster flow velocities will correspond to lower densities in the scattering region. The mass loading of the jet should also affect the asymptotic Lorentz factor of the flow downstream if the jet is magnetically driven (e.g., Vlahakis \& Königl 2004). The same decrease in electron number that causes a drop in scattered X-ray emission near the disk would lead to a time-delayed increase in the speed of the jet downstream. The increase in flow speed of the jet could form a shock wave, eventually seen as a superluminal radio knot (Gómez et al. 1997). This would then give rise to the dip-ejection sequence discussed above.

The highest-amplitude $37 \mathrm{GHz}$ outburst, from 2007.5 to 2008.8 , started at the same time as at $230 \mathrm{GHz}$, but reached maximum level at a later time than both the $230 \mathrm{GHz}$ and X-ray flares. This can be explained by the larger optical depth at the lower frequencies prior to the $37 \mathrm{GHz}$ peak. The similar amplitudes of the 37 and $230 \mathrm{GHz}$ flares follow the pattern of the synchrotron-loss stage of the shock-in-jet model (Marscher \& Gear 1985), which predicts that, in synchrotron flares produced in the jet, the peak amplitude should stay roughly constant as the emission becomes optically thin at progressively lower frequencies.

The mean time delay between the minimum of the X-ray dips and the time of ejection of the corresponding superluminal knot is $0.15 \pm 0.08 \mathrm{yr}$. The average apparent speed of the moving components with well-determined motions is $3.9 c \pm 0.7 c$. Therefore, a knot moves a distance of $0.18 \pm 0.1 \mathrm{pc}$ in $0.15 \mathrm{yr}$, projected on the plane of the sky. (Here we assume that acceleration of the flow to its terminal velocity occurs over a sufficiently short distance that the time that a moving feature spends in the acceleration zone is short compared with the total transit time to the $43 \mathrm{GHz}$ core.) Since the angle of the jet axis of $3 \mathrm{C} 111$ to the line of sight $\sim 18^{\circ}$, the actual distance traveled by the knot, given by $\beta c \delta \Delta t_{\mathrm{obs}} \Gamma$ is $0.6 \pm 0.3 \mathrm{pc}$. Hence, we derive a distance $0.6 \pm 0.3 \mathrm{pc}$ from the corona or the base of the jet (where the X-rays are produced) to the core seen on the $43 \mathrm{GHz}$ VLBA images. This is one of the few cases where, similar to 3C 120 Chatterjee et al. 2009), we are able to specify the distance between the central engine and mm-wave core in an AGN. 


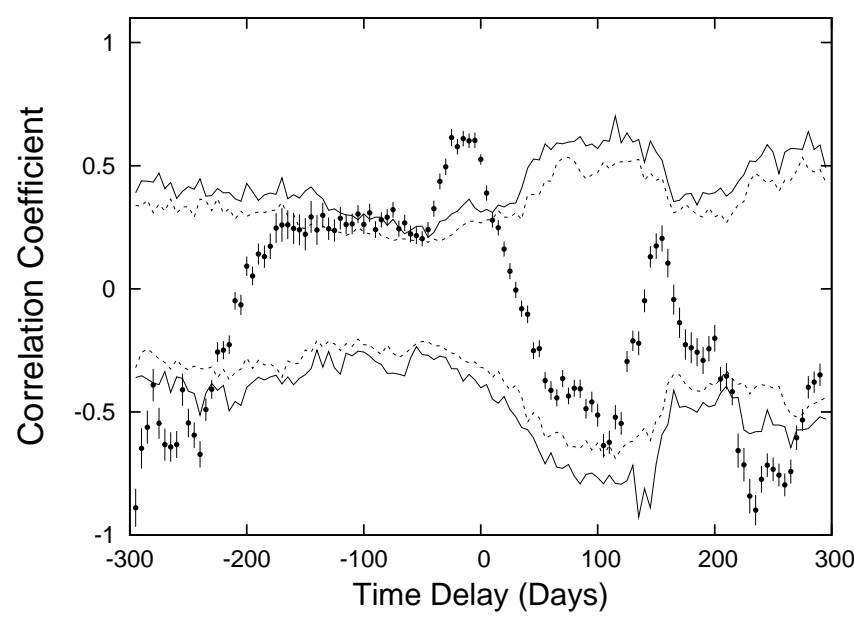

Figure 17. Discrete cross-correlation function (DCCF) of the optical and X-ray monitor data of 3C 111 for the entire $5 \mathrm{yr}$ interval. The time delay is defined as positive if the X-ray variations lag those at the optical frequency. The jagged solid and dotted lines at the top of the panel denote 99 and 95 percent significance levels of correlation (see text), respectively. Those at the bottom of the panel show the same for anti-correlation. The peak X-ray versus optical DCCF is 0.6 , which corresponds to more than $99 \%$ significance level. The peak at -15 days indicates that the X-ray variations lead those in the optical by $15 \pm 10$ days.

\section{X-RAY/OPTICAL CORRELATION}

As determined by the DCCF (Figure 17), we find that the X-ray variations of $3 \mathrm{C} 111$ are very strongly correlated with those at optical $R$ band. The peak X-ray versus optical DCCF is 0.6 , which corresponds to more than $99 \%$ significance level. The jagged solid and dotted lines at the top of the panel denote 99 and 95 percent significance levels of correlation, respectively. Those at the bottom of the panel show the same for anti-correlation, as described in the previous section. The time lag of the peak indicates that the X-ray variations lead those in the optical by $15 \pm 10$ days. The highly significant correlation between the X-ray and optical variations implies that emission at these wave bands is causally connected.

Fig. [5 shows the variation of the degree of polarization as well as the electric vector position angle (EVPA) of 3C 111 at $R$ band from 2006 to the present. The average polarization was only $1.6 \% \pm 0.6 \%$, rather low for synchrotron radiation from the jet. This agrees with the idea that most of the optical emission is thermal radiation from the accretion disk (Malkan 1983). The strong $\mathrm{X}$-ray/optical correlation, weak optical polarization, and smaller variance of optical compared with X-ray flux at shorter timescales are consistent with a reprocessing model. In this scenario, the X-rays are predominantly produced by inverse Compton (IC) scattering of thermal optical/UV seed photons from the accretion disk by hot, but non-relativistic, electrons in the corona, while a significant fraction of the optical-UV emission is due to heating of the accretion disk by X-rays produced in the above process. The slope of the optical PSD in the range $10^{-8.1}$ to $10^{-5.8} \mathrm{~Hz}$ is $\sim-2.0$, significantly steeper than that of the X-ray PSD over the same frequency range $(\sim$ -1.0 ). This implies that the optical variations on shorter time-scales are suppressed, consistent with smoothing by reprocessing.

Alternatively, a substantial fraction of the optical emis- sion may be generated as synchrotron radiation in the jet, giving rise to a degree of polarization up to $3 \%$ (Fig. 5) or even higher (Jorstad et al. 2007) and significant variability of the position angle of polarization (between $0^{\circ}$ and $150^{\circ}$ ) at some epochs. In this case, if X-rays are indeed generated at the base of the jet, then the highly significant correlation between the X-ray and optical variations, combined with the time delay of the latter, $15 \pm 10$ days, implies that the optical emission region in the jet, if present, is situated downstream of the base of the jet. We can calculate the mean distance $(\Delta z)_{\mathrm{xo}}$ between the $\mathrm{X}$-ray and optical synchrotron emission sites in the jet if we adopt $18^{\circ}$ as the angle between the jet axis and the line of sight (Jorstad et al. 2005) and assume a value for the average speed $\beta c$ of the jet flow between the two emission regions: $(\Delta z)_{\mathrm{xo}} \sim 6,20$, and 200 light-days for $\beta=0.25,0.5$, and 0.974 , respectively. The last value, $\beta=0.974$, corresponds to the velocity obtained from analysis of the superluminal apparent motion and timescale of flux decline at $43 \mathrm{GHz}$ of knots observed with the VLBA (Jorstad et al. 2005). The lower values of $\beta$ near the base of the jet are in concert with theoretical models in which jets are accelerated to relativistic flow velocities over extended distances from the central engine (e.g., Vlahakis \& Königl 2004).

Some of the thermal optical-UV emission could be produced in the accretion disk even without reprocessing via $\mathrm{X}$-ray heating. The temporal nature of this emission would be different from that generated as a result of reprocessing. The X-rays may be produced mainly by up-scattering of UV rather than optical photons. This could occur if the flux of optical photons reaching the corona is much smaller than that of UV photons. Such a scenario is likely if the corona is small, such that the UV emission region is much closer to the corona than the region where the majority of the optical photons are produced (see Chatterjee et al. 2009, for details). Any disturbance propagating outward in the accretion disk will cause a change in the UV flux (and a resultant nearly immediate change in the X-ray emission) followed by a similar change in the optical flux. This may also give rise to the X-ray/optical time delay that is observed. As discussed in detail by Chatteriee et al. (2009), the variability timescale described above is consistent with the model proposed by King et al. (2004) and Livio, Pringle \& King (2003), in which variability in the disk emission is caused by large-scale alignment of poloidal magnetic field in the inner accretion disk from random fluctuations in field direction. Such alignment occurs on a timescale of a few tens of days for a $\mathrm{BH}$ of mass of $\sim 10^{8} \mathrm{M}_{\odot}$, consistent with the results described above.

The time delay of $15 \pm 10$ days of the optical with respect to the X-ray variations is larger than expected from a pure reprocessing model. As discussed in Chatteriee et al. (2009), the region of the accretion disk that produces the largest amount of direct (rather than reprocessed) optical emission is at $\sim 100$ gravitational radii $\left(r_{g}\right)$ from the center, which is equal to $\sim 1$ lightday for a BH mass of $1.8 \times 10^{8} \mathrm{M}_{\odot}$ (see also the detailed modeling of Kazanas \& Nayakshin 2001). Therefore, if the X-ray/optical time delay is solely due to light travel time from the corona to the region in the accretion disk 
producing reprocessed optical emission, the corresponding reprocessing region has to be farther and/or extended than this by an order of magnitude $\left(\sim 1000 r_{g}\right)$. If, on the other hand, the variability is caused by disturbances propagating outward through the disk at velocity $\mathrm{v}_{\text {dist }}$, then the optical emission region could be closer by a factor $\mathrm{v}_{\text {dist }} / \mathrm{c}$. If a fraction of the optical emission is produced in the jet, as discussed above, or the variability is partly affected by disturbances propagating in the accretion disk, then the observed time delay and flat peak of the X-ray/optical cross-correlation function could result.

The accretion rate estimated in $\S 3, \dot{m}=$ $(0.02 \pm 0.01) \dot{m}_{\text {edd }}$, is within the range at which the transition from high to low state occurs in the Galactic BHXRBs (Maccarone 2003). An advection dominated accretion flow (ADAF) solution may exist for accretion onto black holes at this rate (Naravan 2005). The radiation in an ADAF is dominated by thermal comptonization, which has been used to explain the X-ray spectra of BHXRBs in the canonical low-hard state (e.g., Esin et al. 1998). It is possible that the $\mathrm{X}$-rays from 3C 111 that we have attributed to a distribution of hot electrons consistent with the corona might instead be produced by an ADAF.

\section{COMPARISON WITH THE FR I RADIO GALAXY 3C 120}

Although 3C 111 and 3C 120 fall into two different Fanaroff-Riley categories, they have a number of common characteristics. Both are radio-loud AGNs with jets that dominate the emission at radio frequencies, but with less radiative power than typical blazars and directed at a wider angle to our line of sight. In both radio galaxies, the low optical polarization is consistent with most of the optical continuum resulting from blackbody radiation from the accretion disk. The X-ray properties are consistent with inverse Compton scattering of thermal optical-UV photons from the disk by a distribution of hot electrons - the corona, which could be the base of the jet - situated near the disk. In 3C 111, some of the optical and X-ray emission could be from the jet. A major difference between these two radio galaxies is in the accretion rate. In $3 \mathrm{C} 120$, the accretion rate is high $\left(\sim 0.3 \dot{m}_{\text {edd }}\right)$, similar to Galactic BHXRBs in the X-ray high state. In 3C 111, it is lower by an order of magnitude ( $\left.\sim 0.02 \dot{m}_{\text {edd }}\right)$ which is close to the transition from high to low state in galactic BHXRBs. Therefore, the inner accretion flow in 3C 111 may be advection dominated.

The X-ray emission from each of these radio galaxies possesses a characteristic timescale that is proportional to the BH mass, similar to what is observed in Galactic BHXRBs and Seyfert galaxies. This result implies that there is a universality in the accretion processes of $\mathrm{BHs}$ in the mass range $10 M_{\odot}$ to $10^{8} M_{\odot}$. These objects also show a clear connection between the radiative state near the $\mathrm{BH}$ and events in the jet. Ejection of new knots in the jet following dips in the X-ray light curve observed in these two radio galaxies is somewhat similar to the Galactic micro-quasar GRS 1915+105, where knots with apparent superluminal motion appear in the jet after the X-ray flux recovers from intervals of very low flux levels (Mirabel et al. 1998). This connection is expected according to current theories of jet launching and collimation, but has not been demonstrated previously to extend to radio-loud AGNs.

The relationships that we have uncovered between the central engine and the jet provide strong support for the paradigm (e.g., Körding. Jester \& Fender 2006; Marecki \& Swoboda 2011) that AGNs and Galactic BHXRBs are fundamentally similar, with characteristic time and size scales proportional to the mass of the central BH. This implies that we can develop and test models of AGNs based partly on observations of BHXRBs.

\section{SUMMARY AND CONCLUSIONS}

This paper presents well-sampled, 6-yr-long light curves of 3C 111 between 2004 and 2010 at X-ray, optical, and radio wavebands, as well as monthly images obtained with the VLBA at $43 \mathrm{GHz}$. The appearance of new knots in the VLBA images of the jet follow prominent dips in the smoothed X-ray light curve, demonstrating a connection between events in the jet at parsec scales and those occurring near or in the accretion disk. We have calculated the broad-band X-ray power spectral density (PSD) of 3C 111 and cross-correlated the X-ray and optical light curves, determining the significance of the correlations with light curves simulated in accordance with the PSD. From the analysis of the temporal properties of the observed variability in multiple wave bands, we infer the locations and radiative mechanisms of the X-ray and optical emission.

Our main conclusions are as follows:

(1) The X-ray PSD of 3C 111 is best fit by a bending power-law model with a smooth change in slope above a break frequency. The best-fit value of the break frequency is $10^{-6.05} \mathrm{~Hz}$, corresponding to a break timescale of 13 days, which agrees with the empirical relation between break timescale. $\mathrm{BH}$ mass, and accretion rate obtained by McHardy et al. (2006) based on a sample of mainly radio-quiet Seyfert galaxies. This indicates that the accretion process in $3 \mathrm{C} 111$ is similar to that of BHXRBs and Seyfert galaxies, and that there is a possible universality in the accretion processes in BHs spanning a broad range of masses from $10 M_{\odot}$ to $10^{8}$ $M_{\odot}$.

(2) In $3 \mathrm{C} 111$, the $2-10 \mathrm{keV}$ continuum flux and the Fe line intensity are strongly correlated, with a time lag shorter than 90 days and consistent with zero. This implies that the $\mathrm{Fe}$ line is generated within 90 light-days of the source of the X-ray continuum.

(3) All X-ray dips are followed by the ejection of a new knot in the VLBA images. We derive a distance from the base of the jet or corona (where the X-rays are produced) to the VLBA $43 \mathrm{GHz}$ core region of $\sim 0.6 \mathrm{pc}$ using the average time delay between the minimum of the X-ray dips and the time of passage of the corresponding superluminal knots through the core. (4) The dip-ejection connection can be explained if a decrease in the X-ray production is linked with increased speed in the jet flow, causing a shock front to eventually form and move downstream. This property of 3C 111 is similar to $3 \mathrm{C} 120$ and reminiscent of Galactic $\mathrm{BH}$ systems, where transitions to high-soft X-ray states are associated with the emergence of very bright features that proceed to propagate down the radio jet.

(5) Most of the optical emission in 3C 111 is generated 
in the accretion disk due to X-ray heating. However, weak optical polarization indicates that some fraction of the optical emission is synchrotron radiation from the jet.

\section{ACKNOWLEDGMENTS}

The research at Boston University (BU) was funded in part by the National Science Foundation (NSF) through grants AST-0406865 and AST-0907893 and by NASA through Astrophysical Data Analysis Program grant NNX08AJ64G and XMM-Newton grant NNX08AY06G. The PRISM camera at Lowell Observatory was developed by $K$. Janes et al. at $\mathrm{BU}$ and Lowell Observatory, with funding from the NSF, BU, and Lowell Observatory. The University of Michigan Radio Astronomy Observatory is supported by funds from the NSF, NASA, and the University of Michigan. The VLBA is an instrument of the National Radio Astronomy Observatory, a facility of the National Science Foundation operated under cooperative agreement by Associated Universities, Inc. The Liverpool Telescope is operated on the island of La Palma by Liverpool John Moores University in the Spanish Observatorio del Roque de los Muchachos of the Instituto de Astrofisica de Canarias, with financial support from the UK Science and Technology Facilities Council. The Metsähovi team acknowledges the support from the Academy of Finland. The research at IAA-CSIC is supported by the Spanish Ministry of Science and Innovation grants AYA2007-67627- C03-03 and AYA2010-14844, and by the Regional Government of Andalucía (Spain) grant P09-FQM-4784. This paper is partly based on observations carried out at the German-Spanish Calar Alto Observatory, which is jointly operated by the MPIA and the IAA-CSIC. Acquisition of the MAPCAT data is supported in part by MICIIN (Spain) grants AYA2007-67627-C03-03 and AYA2010-14844, and by CEIC (Andalucía) grant P09-FQM-4784. The Submillimeter Array is a joint project between the Smithsonian Astrophysical Observatory and the Academia Sinica Institute of Astronomy and Astrophysics and is funded by the Smithsonian Institution and the Academia Sinica.

\section{REFERENCES}

Abdo, A., et al. 2010, ApJS, 188, 405

Aller, H. D., Aller, M. F., Latimer, G. E., \& Hodge, P. E. 1985, ApJS, 59, 513

Baars, J. W. M., Genzel, R., Pauliny-Toth, I. I. K., \& Witzel, A. 1977, A\&A, 61, 99

Begelman, M. C. 1995, Proc. NAS, 92, 11442

Blandford, R. D., \& Payne, D. G. 1982, MNRAS, 199, 883

Chatterjee, R., et al. 2008, ApJ, 689, 79

Chatterjee, R., et al. 2009, ApJ, 704, 1689

D'Arcangelo, F. D., et al. 2007, ApJ, 659, L107

Decarli, R., Dotti, M., \& Treves, A. 2011, MNRAS, in press (arXiv:1011.5879)

Edelson, R. A., \& Krolik, J. H. 1988, ApJ, 333, 646

Edelson, R. A., \& Nandra K. 1999, ApJ, 514, 682

Edelson, R. A., et al. 2002, ApJ, 568, 610

Elvis, M., et al. 1994, ApJ

Eracleous, M. \& Halpern, J. P. 2003, ApJ, 599, 886

Eracleous, M., Sambruna, R., \& Mushotzsky, R. F. 2000, ApJ, 537,654

Esin, A. A., Narayan, R., Cui, W., Grove, J. E. and Zhang, S. N. 1998, ApJ, 505, 854
Fanaroff, B. L. \& Riley, J. M. 1974, MNRAS, 167, 31

Fender, R. P., Belloni, T. M. \& Gallo, E. 2004, MNRAS, 355, 1105

Fender, R. P., Homan, J. \& Belloni, T. M. 2009, MNRAS, 396, 1370

Gaskell, C. M., \& Peterson, B. M. 1987, ApJS, 65, 1

Gómez, J. L., Marti, J. M. A., Marscher, A. P., Ibanez, J. M. A., \& Alberdi, A. ApJ, 482, L33

Götz, M. M. A., Alef, W., Preuss, E., \& Kellermann, K. I. 1987, A\&A, 176,171

Greene, J. E., \& Ho, L. C. 2005, ApJ, 630, 122

Gurwell, M. A., Peck, A. B., Hostler, S. R., Darrah, M. R., \& Katz, C. A. 2007, From Z-Machines to ALMA: (Sub)Millimeter Spectroscopy of Galaxies ASP Conf. Ser., 375, 234

Haardt, F., Maraschi, L., \& Ghisellini, G. 1994, ApJ, 432, L95

Hartman, R. C., Kadler, M., \& Tueller, J. 2008, ApJ, 688, 852

Jorstad, S. G., et al. 2005, AJ, 130, 1418

Jorstad, S. G., et al. 2007, AJ, 134, 799

Kadler, M., et al. 2008, ApJ, 680, 867

Kazanas, D. \& Nayakshin, S. 2001, ApJ, 550, 655

King, A. R., Pringle, J. E., West, R. G., \& Livio, M. 2004, MNRAS, 348, 111

Körding, E. G., Jester, S., \& Fender, R. 2006, MNRAS, 372, 1366

Larson, K. A., Whittet, D. C. B., \& Hough, J. H. 1996, ApJ, 472, 755

Lewis, K. T., Eracleous, M., Gliozzi, M., Sambruna, R. M., \&

Mushotzky, R. F. 2005, ApJ, 622, 816

Linfield, R. \& Perley, R. 1984, ApJ, 279, 60

Livio, M., Pringle, J. E., \& King, A. R., 2003, ApJ, 593184

Lovelace, R. V. E., Berk, H.L., \& Contopoulos, J. 1991, ApJ, 379, 696

Lusso et al. 2010, A\&A, 512, A34

Maccarone, T. J. 2003, A\&A, 409, 697

Malkan, M. A. 1983, ApJ, 268, 582

Marchesini, D., Celotti, A., \& Ferrarese, L. 2004, MNRAS, 351, 733

Marecki, A. \& Swoboda, B. 2011, A\&A, 525, A6

Markoff, S., Nowak, M. A., \& Wilms, J. 2005, ApJ, 635, 1203

Markowitz, A., et al. 2003, ApJ, 593, 96

Markowitz, A., Edelson, R. \& Vaughan, S. 2003, ApJ, 598, 935

Markowitz, A., Reeves, J.N., George, I.M., et al. 2009, ApJ, 691, 922

Marscher, A. P., \& Gear, W. K. 1985, ApJ, 298, 114

Marscher, A. P., \& Jorstad, S. G. 2010, in Fermi meets Jansky AGN at Radio and Gamma-Rays, ed. Savolainen, T., Ros, E. Porcas, R. W., \& Zensus, J. A. (Bonn: MPIfR), 171

Marscher, A. P., Moore, E. M., \& Bania, T. M. 1993, ApJ, 419, L101

Marscher et al. 2002, Nature, 417, 625, 2002

Marshall, K., Ryle, W. T., Miller, H. R., Marscher, A. P., Jorstad, S. G., Chicka, B., \& McHardy, I. M. 2009, ApJ, 696, 601

McHardy, I. M., Koerding, E., Knigge, C., Uttley, P., \& Fender, R. P. 2006, Nature, 444, 730

McHardy I. M., Papadakis I. E., Uttley P., Page M. J., \& Mason K. O. 2004, MNRAS, 348, 783

Meier, D. L., Koide, S., \& Uchida, Y. 2001, Science, 291, 84

Mirabel, I. F., Dhawan, V., Chaty, S., Rodriguez, L. F., Marti, J.,

Robinson, C. R., Swank, J., \& Geballe, T. 1998, A\&A, 330, L9

Moore, E. M., \& Marscher, A. P. 1995, ApJ, 452, 671

Narayan, R. 2005, Ap\&SS, 300, 177

Nandra, K., George, I. M., Mushotzky, R. F., Turner, T. J., \& Yaqoob, T. 1997, ApJ, 476, 70

Nayakshin, S. 200, ApJ, 534, 718

Nowak, M. A., et al. 1999, ApJ, 510, 874

Papadakis, I. E., Petrucci, P. O., Maraschi, L., McHardy, I. M., Uttley, P., \& Haardt, F. 2002, ApJ, 573, 92

Peterson, B. M., Wanders, I., Horne, K., Collier, S., Alexander, T., Kaspi, S., \& Maoz, D. 1998, PASP, 110, 660

Pounds, K. A., Edelson, R. A., Markowitz, A., \& Vaughan, S. 2001, ApJ, 550, L15

Rachford, B. L., et al. 2002, ApJ, 577, 221

Preuss, E., Alef, W., \& Kellermann, K. I. 1987, IAU Symp. 129, ed. M. J. Reid \& J. M. Moran, 105

Remillard, R. A., \& McClintock, J. E. 2006, ARA\&A, 44, 49

Reynolds, C. S., et al. 1998, MNRAS, 299, 410

Rivers, E., Markowitz, A. G., \& Rothschild, R. E. 2011, ApJS, 193,3 
Sambruna, R. M., Eracleous, M., \& Mushotzky, R. F. 1999, ApJ, $526,60 \mathrm{~S}$

Sargent, W. L. W. 1977, ApJ, 212, L105

Schlegel, D. J., Finkbeiner, D. P., \& Davis, M. 1998, ApJ, 500, 525

Sguera, V., Bassani, L., Malizia, A., Dean, A. J., Landi, R., \& Stephen, J. B. 2005, ApJ, 430, 107

Shakura, N. F. \& Sunyaev, R. A. 1973, A\&AS, 24, 337

Shepherd, M. C. 1997, in ASP Conf. Proc. 125, Astronomical Data Analysis Software and Systems VI, ed. G. Hunt \& H. E. Payne (San Francisco: ASP), 77

Shih, D. C., Iwasawa, K., \& Fabian, A. C. 2002, MNRAS, 333, 687

Teräsranta, H. et al. 1998, A\&AS, 132, 305

Ulvestad, J. S., et al. 1999, ApJL, 517, L81
Ungerer, V , et al. 1985, A\&A, 146, 123

Uttley, P., McHardy, I. M., \& Papadakis, I. E. 2002, MNRAS, 332,231

Valtaoja E., et al. 1999, ApJS, 120, 95

Vaughan, S. \& Edelson, R. 2001, ApJ, 548, 694

Vaughan, S., Edelson, R., Warwick, R. S., \& Uttley, P. 2003, MNRAS, 345, 1271

Vestergaard, M. \& Peterson, B. M. 2006, ApJ, 641, 689

Vlahakis, N. \& Königl, A. 2004, ApJ, 605, 656

Young, M., Elvis, M., \& Risaliti, G. 2010, ApJ, 708, 1388

Wardle, J. F. C., \& Kronberg, P. P. 1974, ApJ, 194, 249

White, R. \& Peterson, B.M. 1994, PASP, 106, 879 